\title{
A Two-way Paraxial System for Simulation of Wave Backscattering by a Random Medium
}

\author{
Josselin Garnier \\ Laboratoire de Probabilités et Modèles Aléatoires 85 Laboratoire Jacques-Louis \\ Lions, Université Paris VII, 2 Place Jussieu, 75251 Paris Cedex 05, France. \\ Knut Sølna \\ Department of Mathematics, University of California, Irvine CA 92697
}

\begin{abstract}
This paper presents a probabilistic analysis of an iterative two-way paraxial scheme for the simulation of wave propagation in random media. This scheme has the computational cost of the standard one-way paraxial wave equation but has the accuracy of the full wave equation in a regime beyond the classical paraxial regime. More precisely, it accurately predicts the statistics of the reflected wave field. The accuracy is determined by the order of the iterative scheme and the ratio of the random backscattering intensity over the random forward-scattering intensity.

Keywords: numerical wave propagation, parabolic approximation, random media, diffusion approximation
\end{abstract}

MSC: 65C20, 74J20, 35R60

\section{Introduction}

Wave propagation in random media is numerically challenging when the propagation distance is large compared to the wavelength. The resolution of the full wave equation by difference methods is then in general computationally expensive since relatively fine scales have to be resolved. Fortunately, in many applications, such as ocean acoustics or atmospheric beam propagation, backscattering is negligible and the wave propagates mainly in a privileged direction, which allows the reduction of the wave equation to the paraxial (or one-way)

Email addresses: garnier@math.jussieu.fr (Josselin Garnier), ksolna@math.uci.edu (Knut Sølna).

Preprint submitted to Elsevier Science 10 June 2008 
wave equation [15]. The time harmonic form of this equation takes the form of a Schrödinger equation with a random potential which makes it easy to solve numerically (by finite-difference or split-step Fourier methods) and allows for an easy theoretical analysis from the statistical point of view, since the solution is adapted to the filtration of the process that describes the fluctuations of the random medium. The spatial argument corresponding to the privileged direction of propagation plays the role of "time" in the Schrödinger equation and also for the filtration of the medium fluctuations process. In this paper we address situations in which backscattering is not negligible, which requires more elaborate schemes than the paraxial wave equation. After the pioneering work [5] an iterative two-way paraxial scheme was proposed to solve the full wave equation by an iteration of forward-going and backward-going paraxial wave equations in $[12,13]$. Comparisons between numerical simulations of the full wave and numerical simulations of the two-way paraxial system have shown very good agreement, with the overall conclusion being that the twoway paraxial scheme has the same numerical complexity as the standard oneway paraxial equation, but it also takes into account random backscattering. However, theoretical justifications are yet to be found. In particular, the role of the order of the iterative scheme is not clear in these first papers.

The purpose of this paper is to give theoretical arguments that show that the two-way paraxial system can indeed give accurate predictions for the transmitted and reflected waves in propagation regimes in which backscattering due to random medium fluctuations is significant. The general motivation for numerical wave propagation in random media is to examine the effects that medium heterogeneity (which is very complicated and cannot easily be identified explicitly) has on the propagating wave. The motivation may for instance be to analyze the role of fine scale medium fluctuations in the context of imaging schemes aimed at identifying macroscale features of the medium. What is relevant in this context is therefore the law or statistics of transmitted and reflected wave fields. It is important to note that our goal is thus not to give arguments for a strong equivalence between the paraxial system and the wave equation, in the sense that the wave fields obtained from the two systems coincide (with some accuracy) for a given realization of the random medium. Rather, we seek to show that it is possible to prove a weak equivalence, in the sense that the statistics of the wave fields obtained from the two systems coincide. As expected, the degree of accuracy of the two-way iterative scheme depends on the order of the scheme (i.e. the number of iterations) and we clarify the connection between accuracy and order. We focus in particular our attention on the first-order and the second-order two-way paraxial equation for the reflected wave field and some associated applications.

The paper is organized as follows. In Section 2 we present the full wave equation in random medium and its reduction to the standard paraxial wave equation. In Section 3 we introduce the two-way scheme. The statistical analysis 
of the reflected wave field is carried out in Section 5. This analysis requires us to transform the boundary value problem satisfied by the reflected and transmitted wave fields into an initial value problem by an invariant imbedding technique which is presented in Section 4.

\section{The Wave Decomposition}

We consider linear acoustic waves propagating in a $1+d$-dimensional heterogeneous medium. The governing equations for the pressure field $p$ and the velocity field $\mathbf{u}$ are

$$
\rho(z, \mathbf{x}) \frac{\partial \mathbf{u}}{\partial t}+\nabla p=\mathbf{0}, \quad \frac{1}{K(z, \mathbf{x})} \frac{\partial p}{\partial t}+\nabla \cdot \mathbf{u}=0
$$

where $\rho$ is the density of the medium, $K$ is the bulk modulus of the medium, and $(z, \mathbf{x}) \in \mathbb{R} \times \mathbb{R}^{d}$ are the space coordinates. We assume that a section of heterogeneous medium is sandwiched in between two homogeneous half-spaces and we consider the following model for the bulk modulus and density

$$
\begin{aligned}
& \frac{1}{K(z, \mathbf{x})}= \begin{cases}K_{0}^{-1} & \text { if } z \in(-\infty, 0) \cup(L, \infty) \\
K_{0}^{-1}(1+\nu(z, \mathbf{x})) & \text { if } z \in(0, L)\end{cases} \\
& \rho(z, \mathbf{x})=\rho_{0}
\end{aligned}
$$

where $K_{0}$ and $\rho_{0}$ are two positive constants and $\nu(z, \mathbf{x})$ is a random field that is bounded by a constant smaller than one. The acoustic wave equations can then be reduced to the inhomogeneous wave equation for the pressure field:

$$
\Delta p-\frac{\rho_{0}}{K(z, \mathbf{x})} \frac{\partial^{2} p}{\partial t^{2}}=0
$$

where $\Delta$ is the $1+d$-dimensional Laplacian. By taking a Fourier transform with respect to time

$$
\check{p}(\omega, z, \mathbf{x})=\int p(t, z, \mathbf{x}) e^{i \omega t} d t, \quad p(t, z, \mathbf{x})=\frac{1}{2 \pi} \int \check{p}(\omega, z, \mathbf{x}) e^{-i \omega t} d \omega
$$

we obtain the inhomogeneous Helmholtz equation

$$
\Delta \check{p}+\left(1+\nu(z, \mathbf{x}) \mathbf{1}_{(0, L)}(z)\right) \frac{\omega^{2}}{c_{0}^{2}} \check{p}=0,
$$

where we have introduced the background velocity $c_{0}=\sqrt{K_{0} / \rho_{0}}$. The Helmholtz equation is complemented by boundary values at the interfaces $z=0$ and 
$z=L$ given by the incoming field and also radiation conditions. These boundary and radiation conditions have a convenient representation if we decompose the solution of the wave equation into generalized right- and left-going mode amplitudes $\check{a}$ and $\check{b}[9,10]$ :

$$
\begin{aligned}
\check{p}(\omega, z, \mathbf{x}) & =\check{a}(\omega, z, \mathbf{x}) e^{i \frac{\omega}{c_{0}} z}+\check{b}(\omega, z, \mathbf{x}) e^{-i \frac{\omega}{c_{0}} z} \\
\frac{\partial \check{p}}{\partial z}(\omega, z, \mathbf{x}) & =\frac{i \omega}{c_{0}}\left(\check{a}(\omega, z, \mathbf{x}) e^{i \frac{\omega}{c_{0}} z}-\check{b}(\omega, z, \mathbf{x}) e^{-i \frac{\omega}{c_{0}} z}\right)
\end{aligned}
$$

The mode amplitudes satisfy the boundary value problem:

$$
\begin{aligned}
& \frac{\partial \check{a}}{\partial z}=\mathcal{L}_{\omega}(z) \check{a}+e^{-2 i \frac{\omega}{c_{0}} z} \mathcal{L}_{\omega}(z) \check{b}, \\
& \frac{\partial \check{b}}{\partial z}=-e^{2 i \frac{\omega}{c_{0}} z} \mathcal{L}_{\omega}(z) \check{a}-\mathcal{L}_{\omega}(z) \check{b},
\end{aligned}
$$

with the boundary conditions

$$
\check{a}(\omega, z=0, \mathbf{x})=0 \quad \text { and } \quad \check{b}(\omega, z=L, \mathbf{x})=\check{b}_{\text {inc }}(\omega, \mathbf{x}),
$$

that correspond to a radiation condition at $z=0$ and an incoming left-going beam at $z=L$. Here we have introduced the operator

$$
\mathcal{L}_{\omega}(z)=\frac{i \omega}{2 c_{0}} \nu(z, \mathbf{x})+\frac{i c_{0}}{2 \omega} \Delta_{\perp}
$$

and $\Delta_{\perp}$ is the transverse $d$-dimensional Laplacian.

The Helmholtz equation (or equivalently the system (4-5)) is a boundary value problem that is not easy to solve in the high-frequency regime, in which the size $L$ of the computational domain is much larger than the typical wavelength of the incoming beam. The paraxial (or forward-scattering) approximation consists in neglecting the backscattered wave $\check{a}$. The transmitted field then satisfies the initial value problem (starting from $z=L$ and going from $L$ to $0)$ :

$$
\frac{\partial \check{b}}{\partial z}=-\mathcal{L}_{\omega}(z) \check{b},
$$

with the initial condition $\check{b}(\omega, z=L, \mathbf{x})=\check{b}_{\text {inc }}(\omega, \mathbf{x})$. The advantage of this simplified equation is that it is easy to solve numerically with a split-step Fourier method or a finite-difference method. It is also possible to study the paraxial wave equation with a random medium since the solution is adapted to the filtration of the random process $\nu$ and stochastic calculus can be applied $[2,4,6-8,14]$. The validity of the paraxial wave equation has been intensively reviewed, and it turns out that the paraxial equation (8) is a good approximation of the Helmholtz equation (3) if the length scale of transverse variations of 
the initial beam $\check{b}_{\text {inc }}$ is larger than the wavelength and if the fluctuations of the medium are also small. Under these conditions, backscattering is indeed small and can be neglected for the computation of the transmitted wave. Our goal is to go beyond the paraxial wave regime and to propose a way to solve the boundary value problem (4-5), which is equivalent to the Helmholtz equation, with a computational cost equivalent to that of the one-way paraxial wave equation. We will show that an iterative two-way paraxial equation can reproduce the results of the full wave equation in a regime where backscattering is significant, which means that this regime is beyond the paraxial regime.

\section{The Two-way Paraxial Wave Equation}

\subsection{Instability of the Iterative Scheme}

We discuss first an idea for solving the system (4-5) with the boundary conditions (6) that corresponds to an iterative scheme in which one solves (4) as an initial-value problem for $\check{a}$ with an approximation for $\breve{b}$ and then one solves (5) as an initial-value problem for $\check{b}$ with an approximation for $\check{a}$. The iteration is expected to converge to a fixed point that should be the desired solution. The two-way paraxial scheme is as follows:

Step 0: start from $\check{a}_{0}(\omega, z, \mathbf{x})=0$ and $\check{b}_{0}(\omega, z, \mathbf{x})=\check{b}_{\text {inc }}(\omega, \mathbf{x})$ for all $z$.

Step $n$ : compute $\check{b}_{n}$ by solving the paraxial wave equation with source from $z=L$ to $z=0$ :

$$
\frac{\partial \check{b}_{n}}{\partial z}=-\mathcal{L}_{\omega}(z) \check{b}_{n}-e^{2 i \frac{\omega}{c_{0}} z} \mathcal{L}_{\omega}(z) \check{a}_{n-1}
$$

starting from $\check{b}_{n}(\omega, z=L, \mathbf{x})=\check{b}_{\text {inc }}(\omega, \mathbf{x})$, and then compute $\check{a}_{n}$ by solving the paraxial wave equation with source from $z=0$ to $z=L$ :

$$
\frac{\partial \check{a}_{n}}{\partial z}=\mathcal{L}_{\omega}(z) \check{a}_{n}+e^{-2 i \frac{\omega}{c_{0}} z} \mathcal{L}_{\omega}(z) \check{b}_{n}
$$

starting from $\check{a}_{n}(\omega, z=0, \mathbf{x})=0$.

Notice that the first-order solution $\check{b}_{1}$ is the transmitted wave in the standard paraxial (or forward-scattering) approximation (since $\check{a}_{0}=0$ ). The scheme (910) seems natural: it iteratively computes the transmitted $\left(\breve{b}_{n}\right)$ and backscattered $\left(\check{a}_{n}\right)$ waves, and if the pair of functions $\left(\check{a}_{n}, \check{b}_{n}\right)$ were converging, then it would converge to a fixed point that would be the solution of the system (4-5). The problem is that the scheme (9-10) is unstable when the distance $L$ is larger than the wavelength, which is the high-frequency regime of interest in most applications. This instability was first noticed in [12]. It is due to the cross transverse Laplacian as shown by the following lemma. 
Lemma 3.1 Let us assume that $\nu=0$ and let us consider the transverse Fourier transforms of the functions $\check{a}_{n}$ and $\check{b}_{n}$ :

$$
\hat{a}_{n}(\omega, z, \boldsymbol{\kappa})=\int \check{a}_{n}(\omega, z, \mathbf{x}) e^{-i \boldsymbol{\kappa} \cdot \mathbf{x}} d \mathbf{x}, \quad \hat{b}_{n}(\omega, z, \boldsymbol{\kappa})=\int \check{b}_{n}(\omega, z, \mathbf{x}) e^{-i \boldsymbol{\kappa} \cdot \mathbf{x}} d \mathbf{x} .
$$

If $|\boldsymbol{\kappa}|^{2}=2 \omega^{2} / c_{0}^{2}$ and $\omega L / c_{0}>\pi / 2$, then $\hat{b}_{n}(\omega, 0, \boldsymbol{\kappa})$ diverges exponentially as $n \rightarrow \infty$ as

$$
\left|\hat{b}_{n}(\omega, 0, \kappa)\right| \simeq \frac{4}{\pi}\left(\frac{2 \omega L}{\pi c_{0}}\right)^{2 n-2}\left|\hat{b}_{\mathrm{inc}}(\omega, \boldsymbol{\kappa})\right|
$$

Proof. Notice first that now

$$
\frac{\partial \hat{a}_{n}}{\partial z}=-\frac{i c_{0}|\kappa|^{2}}{2 \omega}\left(\hat{a}_{n}+e^{-2 i \frac{\omega}{c_{0}} z} \hat{b}_{n}\right), \quad \frac{\partial \hat{b}_{n}}{\partial z}=\frac{i c_{0}|\kappa|^{2}}{2 \omega}\left(\hat{b}_{n}+e^{2 i \frac{\omega}{c_{0}} z} \hat{a}_{n}\right) .
$$

Let us thus fix a transverse wavevector $\boldsymbol{\kappa}$ such that $|\boldsymbol{\kappa}|^{2}=2 \omega^{2} / c_{0}^{2}$. The rescaled functions

$$
\tilde{a}_{n}(\tilde{z})=i \frac{\hat{a}_{n}\left(\omega, \frac{c_{0}}{\omega} \tilde{z}, \boldsymbol{\kappa}\right)}{\hat{b}_{\text {inc }}(\omega, \boldsymbol{\kappa})} e^{i z}, \quad \tilde{b}_{n}(\tilde{z})=\frac{\hat{b}_{n}\left(\omega, \frac{c_{0}}{\omega} \tilde{z}, \boldsymbol{\kappa}\right)}{\hat{b}_{\text {inc }}(\omega, \boldsymbol{\kappa})} e^{-i \tilde{z}}
$$

then satisfy the system

$$
\frac{d \tilde{b}_{n}}{d \tilde{z}}=\tilde{a}_{n-1}, \quad \tilde{b}_{n}(\tilde{L})=1, \quad \frac{d \tilde{a}_{n}}{d \tilde{z}}=\tilde{b}_{n}, \quad \tilde{a}_{n}(0)=0
$$

where $\tilde{L}=\omega L / c_{0}$. Note that the correspondingly scaled transverse Fourier transform of the solution of the boundary value problem (4-5) satisfies

$$
\frac{d \tilde{b}}{d \tilde{z}}=\tilde{a}, \quad \tilde{b}(\tilde{L})=1, \quad \frac{d \tilde{a}}{d \tilde{z}}=\tilde{b}, \quad \tilde{a}(0)=0 .
$$

It is a pair of smooth functions bounded by one:

$$
\tilde{a}(\tilde{z})=\frac{\sinh (\tilde{z})}{\cosh (\tilde{L})}, \quad \tilde{b}(\tilde{z})=\frac{\cosh (\tilde{z})}{\cosh (\tilde{L})}
$$

The solution $\left(\tilde{a}_{n}, \tilde{b}_{n}\right)$ of the two-way scheme does not always converge to $(\tilde{a}, \tilde{b})$. Indeed, we can show by induction that the pair of functions $\left(\tilde{a}_{n}, \tilde{b}_{n}\right)$ is given by

$\tilde{a}_{n}(\tilde{z})=\sum_{j=0}^{n-1} \sum_{l=0}^{n-1-j} \frac{E_{2 l}}{(2 j+1) !(2 l) !} \tilde{L}^{2 l} \tilde{z}^{2 j+1}, \quad \tilde{b}_{n}(\tilde{z})=\sum_{j=0}^{n-1} \sum_{l=0}^{n-1-j} \frac{E_{2 l}}{(2 j) !(2 l) !} \tilde{L}^{2 l} \tilde{z}^{2 j}$, 
where the $E_{2 l}$ 's are the Euler numbers, which are associated to the series expansion [1, formula 4.5.66]

$$
\frac{1}{\cosh (\tilde{L})}=\sum_{l=0}^{\infty} \frac{E_{2 l}}{(2 l) !} \tilde{L}^{2 l}
$$

and whose behavior is [1, formula 23.1.15]

$$
\frac{2^{2 l+2}}{\pi^{2 l+1}} \frac{1}{1+3^{-1-2 l}}<(-1)^{l} \frac{E_{2 l}}{(2 l) !}<\frac{2^{2 l+2}}{\pi^{2 l+1}} .
$$

In particular,

$$
\tilde{b}_{n}(0)=\sum_{l=0}^{n-1} \frac{E_{2 l}}{(2 l) !} \tilde{L}^{2 l}
$$

is an alternating series whose general term is equivalent to $(-1)^{l} \frac{4}{\pi}\left(\frac{2 \tilde{L}}{\pi}\right)^{2 l}$. Consequently, the series is divergent if $\frac{2 \tilde{L}}{\pi}>1$.

\subsection{The Corrected Iterative Scheme}

We now propose a corrected scheme that avoids the previous instability. We introduce the operators

$$
\mathcal{S}_{\omega}^{ \pm}(z)=e^{ \pm 2 i \frac{\omega}{c_{0}} z} \frac{i \omega}{2 c_{0}} \nu(z, \mathbf{x})
$$

and we define the new two-way paraxial scheme as follows:

Step 0: start from $\check{a}_{0}(\omega, z, \mathbf{x})=0$ and $\check{b}_{0}(\omega, z, \mathbf{x})=\check{b}_{\text {inc }}(\omega, \mathbf{x})$ for all $z$.

Step $n$ : compute $\breve{b}_{n}$ by solving the paraxial wave equation with source from $z=L$ to $z=0$ :

$$
\frac{\partial \check{b}_{n}}{\partial z}=-\mathcal{L}_{\omega}(z) \check{b}_{n}-\mathcal{S}_{\omega}^{+}(z) \check{a}_{n-1}
$$

starting from $\check{b}_{n}(\omega, z=L, \mathbf{x})=\check{b}_{\text {inc }}(\omega, \mathbf{x})$, and then compute $\check{a}_{n}$ by solving the paraxial wave equation with source from $z=0$ to $z=L$ :

$$
\frac{\partial \check{a}_{n}}{\partial z}=\mathcal{L}_{\omega}(z) \check{a}_{n}+\mathcal{S}_{\omega}^{-}(z) \check{b}_{n}
$$

starting from $\check{a}_{n}(\omega, z=0, \mathbf{x})=0$.

We again notice that the first-order solution $\check{b}_{1}$ is the transmitted wave in the standard paraxial (or forward-scattering) approximation. Compared to the scheme (9-10) described in the previous subsection, the crossed terms involving the transverse Laplacian $\exp \left( \pm 2 i \omega z / c_{0}\right) \Delta_{\perp}$ have been removed, while we have kept the crossed terms involving the random process $\nu$. As a consequence, if 
the sequence of functions $\left(\check{a}_{n}, \check{b}_{n}\right)$ converges in a sense strong enough to the pair $\left(\check{a}_{\infty}, \check{b}_{\infty}\right)$ as $n \rightarrow \infty$, then the limit will not satisfy the system (4-5) exactly, because the crossed terms $\exp \left(-2 i \omega z / c_{0}\right) \Delta_{\perp} \check{b}$ in (4) and $\exp \left(2 i \omega z / c_{0}\right) \Delta_{\perp} \check{a}$ in (5) will be absent. Therefore, the iterative scheme cannot give the exact result of the full wave equation, even in the limit $n \rightarrow \infty$. However, the main goal of this paper is to show that the iterative scheme is a good approximation of the full wave equation in a specific regime (in which the crossed terms associated with the transverse Laplacian are indeed negligible, while random backscattering is significant).

We first give an elementary lemma for the convergence of the two-way paraxial scheme with a strong hypothesis on the amplitude of the random fluctuations. The proof of the lemma is given in Appendix A.

Lemma 3.2 If $\|\nu\|_{\infty}$ is small enough (a sufficient condition is $\omega\|\nu\|_{\infty} L<$ $\left.2 c_{0} / \sqrt{3}\right)$, then the pair of functions $\left(\check{a}_{n}, \check{b}_{n}\right)$ converges exponentially in $L^{\infty}\left([0, L], L^{2}\left(\mathbb{R}^{d}\right)\right)$ to the solution of the boundary value problem

$$
\frac{\partial \check{b}_{\infty}}{\partial z}=-\mathcal{L}_{\omega}(z) \check{b}_{\infty}-\mathcal{S}_{\omega}^{+}(z) \check{a}_{\infty}, \quad \frac{\partial \check{a}_{\infty}}{\partial z}=\mathcal{L}_{\omega}(z) \check{a}_{\infty}+\mathcal{S}_{\omega}^{-}(z) \check{b}_{\infty}
$$

with the boundary conditions $\check{a}_{\infty}(\omega, z=0, \mathbf{x})=0$ and $\check{b}_{\infty}(\omega, z=L, \mathbf{x})=$ $\check{b}_{\text {inc }}(\omega, \mathbf{x})$. The limit pair of functions satisfies the conservation of energy relation

$$
\left\|\check{a}_{\infty}(\omega, L, \cdot)\right\|_{2}+\left\|\check{b}_{\infty}(\omega, 0, \cdot)\right\|_{2}=\left\|\check{b}_{\text {inc }}(\omega, \cdot)\right\|_{2},
$$

where $\|\cdot\|_{2}$ stands for the $L^{2}\left(\mathbb{R}^{d}\right)$-norm.

For any $k \geq 1$, if $\nu$ belongs to $L^{\infty}\left([0, L], W^{k, \infty}\left(\mathbb{R}^{d}\right)\right)$ and the corresponding norm is small enough, then the functions $\left(\check{a}_{n}, \check{b}_{n}\right)$ converges exponentially in $L^{\infty}\left([0, L], H^{k}\left(\mathbb{R}^{d}\right)\right)$ to the solution of the boundary value problem (14), provided the initial condition $\check{b}_{\text {inc }} \in H^{k}\left(\mathbb{R}^{d}\right)$.

The conservation of energy relation (15) means that the power of the incoming wave is equal to the sum of the power of the reflected wave $\left(\check{a}_{\infty}(\omega, L, \cdot)\right)$ and the one of the transmitted wave $\left(\check{b}_{\infty}(\omega, 0, \cdot)\right)$.

\section{The Reflection Operator for the Two-way Paraxial Wave Equa- tion}

In the next section we will study the statistics of the reflected wave. The statistical analysis of the solution of the iterative scheme is not easy since we deal with a two-point boundary value problem, so the solution is not adapted

to the filtration of the random field $\nu$. However, it is possible to transform this 
boundary value problem into an initial value problem by using an invariant imbedding technique [3]. This section is devoted to the presentation of this result. We first rewrite the system in the Fourier domain. We consider the transverse Fourier transforms of the modes

$$
\hat{a}_{n}(\omega, z, \boldsymbol{\kappa})=\int \check{a}_{n}(\omega, z, \mathbf{x}) e^{-i \boldsymbol{\kappa} \cdot \mathbf{x}} d \mathbf{x}, \quad \hat{b}_{n}(\omega, z, \boldsymbol{\kappa})=\int \check{b}_{n}(\omega, z, \mathbf{x}) e^{-i \boldsymbol{\kappa} \cdot \mathbf{x}} d \mathbf{x} .
$$

In the Fourier domain the operator $\mathcal{L}_{\omega}(z)$ can be expressed explicitly in terms of the kernel process $\widehat{\mathcal{L}}_{\omega}\left(z, \boldsymbol{\kappa}, \boldsymbol{\kappa}^{\prime}\right)$

$$
\int e^{-i \boldsymbol{\kappa} \cdot \mathbf{x}} \mathcal{L}_{\omega}(z) \psi(\mathbf{x}) d \mathbf{x}=\int \widehat{\mathcal{L}}_{\omega}\left(z, \boldsymbol{\kappa}, \boldsymbol{\kappa}^{\prime}\right) \hat{\psi}\left(\boldsymbol{\kappa}^{\prime}\right) d \boldsymbol{\kappa}^{\prime}
$$

for $\psi$ a test function and where

$$
\widehat{\mathcal{L}}_{\omega}\left(z, \boldsymbol{\kappa}, \boldsymbol{\kappa}^{\prime}\right)=\frac{i \omega}{2(2 \pi)^{d} c_{0}} \hat{\nu}\left(z, \boldsymbol{\kappa}-\boldsymbol{\kappa}^{\prime}\right)-\frac{i c_{0}}{2 \omega}|\boldsymbol{\kappa}|^{2} \delta\left(\boldsymbol{\kappa}-\boldsymbol{\kappa}^{\prime}\right)
$$

Similarly the kernel processes associated with $\mathcal{S}_{\omega}^{ \pm}(z)$ are

$$
\widehat{\mathcal{S}}_{\omega}^{ \pm}\left(z, \boldsymbol{\kappa}, \boldsymbol{\kappa}^{\prime}\right)=e^{ \pm 2 i \frac{\omega}{c_{0}} z} \frac{i \omega}{2(2 \pi)^{d} c_{0}} \hat{\nu}\left(z, \boldsymbol{\kappa}-\boldsymbol{\kappa}^{\prime}\right)
$$

In this framework, the two equations (9-10) of the two-way paraxial system read:

$$
\begin{aligned}
& \frac{\partial \hat{b}_{n}}{\partial z}(\omega, z, \boldsymbol{\kappa}) \\
& \quad=-\int \hat{\mathcal{L}}_{\omega}\left(z, \boldsymbol{\kappa}, \boldsymbol{\kappa}^{\prime}\right) \hat{b}_{n}\left(\omega, z, \boldsymbol{\kappa}^{\prime}\right) d \boldsymbol{\kappa}^{\prime}-\int \hat{\mathcal{S}}_{\omega}^{+}\left(z, \boldsymbol{\kappa}, \boldsymbol{\kappa}^{\prime}\right) \hat{a}_{n-1}\left(\omega, z, \boldsymbol{\kappa}^{\prime}\right) d \boldsymbol{\kappa}^{\prime}
\end{aligned}
$$

starting from $\hat{b}_{n}(\omega, z=L, \boldsymbol{\kappa})=\hat{b}_{\text {inc }}(\omega, \boldsymbol{\kappa})$, and

$$
\begin{aligned}
& \frac{\partial \hat{a}_{n}}{\partial z}(\omega, z, \boldsymbol{\kappa}) \\
& \quad=\int \widehat{\mathcal{L}}_{\omega}\left(z, \boldsymbol{\kappa}, \boldsymbol{\kappa}^{\prime}\right) \hat{a}_{n}\left(\omega, z, \boldsymbol{\kappa}^{\prime}\right) d \boldsymbol{\kappa}^{\prime}+\int \widehat{\mathcal{S}}_{\omega}^{-}\left(z, \boldsymbol{\kappa}, \boldsymbol{\kappa}^{\prime}\right) \hat{b}_{n}\left(\omega, z, \boldsymbol{\kappa}^{\prime}\right) d \boldsymbol{\kappa}^{\prime}
\end{aligned}
$$

starting from $\hat{a}_{n}(\omega, z=0, \boldsymbol{\kappa})=0$.

Using the invariant imbedding technique as explained in Appendix B, we obtain the following result.

Proposition 4.1 The reflected wave predicted by the $n$-th order two-way paraxial wave equation is given by 


$$
\begin{aligned}
p_{\mathrm{ref}}^{(n)}(t, \mathbf{x}) & =\frac{1}{(2 \pi)^{d+1}} \iint \hat{a}_{n}(\omega, L, \boldsymbol{\kappa}) e^{i \boldsymbol{\kappa} \cdot \mathbf{x}} e^{i \frac{\omega}{c_{0}} L} e^{-i \omega t} d \boldsymbol{\kappa} d \omega, \\
\hat{a}_{n}(\omega, L, \boldsymbol{\kappa}) & =\int \widehat{\mathcal{R}}^{(n)}\left(\omega, L, \boldsymbol{\kappa}, \boldsymbol{\kappa}^{\prime}\right) \hat{b}_{\mathrm{inc}}\left(\omega, \boldsymbol{\kappa}^{\prime}\right) d \boldsymbol{\kappa}^{\prime},
\end{aligned}
$$

where $\widehat{\boldsymbol{\mathcal { R }}}^{(n)}$ has the form

$$
\widehat{\mathcal{R}}^{(n)}=\sum_{j=1}^{n} \widehat{\mathcal{R}}_{j},
$$

and the kernels $\widehat{\boldsymbol{\mathcal { R }}}_{j}\left(\omega, z, \boldsymbol{\kappa}, \boldsymbol{\kappa}^{\prime}\right), j=1, \ldots, n$, satisfy the equations

$$
\begin{aligned}
\frac{\partial \widehat{\boldsymbol{\mathcal { R }}}_{1}}{\partial z}= & \int \widehat{\mathcal{L}}_{\omega}\left(z, \boldsymbol{\kappa}, \boldsymbol{\kappa}_{1}\right) \widehat{\mathcal{R}}_{1}\left(\omega, z, \boldsymbol{\kappa}_{1}, \boldsymbol{\kappa}^{\prime}\right)+\widehat{\mathcal{R}}_{1}\left(\omega, z, \boldsymbol{\kappa}, \boldsymbol{\kappa}_{1}\right) \widehat{\mathcal{L}}_{\omega}\left(z, \boldsymbol{\kappa}_{1}, \boldsymbol{\kappa}^{\prime}\right) d \boldsymbol{\kappa}_{1} \\
& +\widehat{\mathcal{S}}_{\omega}^{-}\left(z, \boldsymbol{\kappa}, \boldsymbol{\kappa}^{\prime}\right), \\
\frac{\partial \widehat{\boldsymbol{\mathcal { R }}}_{j}}{\partial z}= & \int \widehat{\mathcal{L}}_{\omega}\left(z, \boldsymbol{\kappa}, \boldsymbol{\kappa}_{1}\right) \widehat{\boldsymbol{\mathcal { R }}}_{j}\left(\omega, z, \boldsymbol{\kappa}_{1}, \boldsymbol{\kappa}^{\prime}\right)+\widehat{\boldsymbol{\mathcal { R }}}_{j}\left(\omega, z, \boldsymbol{\kappa}, \boldsymbol{\kappa}_{1}\right) \widehat{\mathcal{L}}_{\omega}\left(z, \boldsymbol{\kappa}_{1}, \boldsymbol{\kappa}^{\prime}\right) d \boldsymbol{\kappa}_{1} \\
& +\sum_{l=1}^{j-1} \iint \widehat{\boldsymbol{\mathcal { R }}}_{j-l}\left(\omega, z, \boldsymbol{\kappa}, \boldsymbol{\kappa}_{1}\right) \widehat{\mathcal{\mathcal { S }}}_{\omega}^{+}\left(z, \boldsymbol{\kappa}_{1}, \boldsymbol{\kappa}_{2}\right) \widehat{\mathcal{\mathcal { R }}}_{l}\left(\omega, z, \boldsymbol{\kappa}_{2}, \boldsymbol{\kappa}^{\prime}\right) d \boldsymbol{\kappa}_{1} d \boldsymbol{\kappa}_{2},
\end{aligned}
$$

for $j \geq 2$, with the initial conditions $\widehat{\boldsymbol{\mathcal { R }}}_{j}\left(\omega, z=0, \boldsymbol{\kappa}, \boldsymbol{\kappa}^{\prime}\right)=0$ for all $j \geq 1$.

In the same way, we obtain the following proposition describing the transmitted wave.

Proposition 4.2 The transmitted wave predicted by the $n$-th order two-way paraxial wave equation is given by

$$
\begin{aligned}
p_{\mathrm{tr}}^{(n)}(t, \mathbf{x}) & =\frac{1}{(2 \pi)^{d+1}} \iint \hat{b}_{n}(\omega, 0, \boldsymbol{\kappa}) e^{i \boldsymbol{\kappa} \cdot \mathbf{x}} e^{-i \omega t} d \boldsymbol{\kappa} d \omega \\
\hat{b}_{n}(\omega, 0, \boldsymbol{\kappa}) & =\int \widehat{\mathcal{T}}^{(n)}\left(\omega, L, \boldsymbol{\kappa}, \boldsymbol{\kappa}^{\prime}\right) \hat{b}_{\mathrm{inc}}\left(\omega, \boldsymbol{\kappa}^{\prime}\right) d \boldsymbol{\kappa}^{\prime}
\end{aligned}
$$

where $\widehat{\mathcal{T}}^{(n)}$ has the form

$$
\widehat{\mathcal{T}}^{(n)}=\sum_{j=1}^{n} \widehat{\mathcal{T}}_{j}
$$

and the kernels $\widehat{\mathcal{T}}_{j}\left(\omega, z, \boldsymbol{\kappa}, \boldsymbol{\kappa}^{\prime}\right), j=1, \ldots, n$, satisfy the equations 


$$
\begin{aligned}
\frac{\partial \widehat{\mathcal{T}}_{1}}{\partial z}= & \int \widehat{\mathcal{T}}_{1}\left(\omega, z, \boldsymbol{\kappa}, \boldsymbol{\kappa}_{1}\right) \widehat{\mathcal{L}}_{\omega}\left(z, \boldsymbol{\kappa}_{1}, \boldsymbol{\kappa}^{\prime}\right) d \boldsymbol{\kappa}_{1} \\
\frac{\partial \widehat{\mathcal{T}}_{j}}{\partial z}= & \int \widehat{\mathcal{T}}_{j}\left(\omega, z, \boldsymbol{\kappa}, \boldsymbol{\kappa}_{1}\right) \widehat{\mathcal{L}}_{\omega}\left(z, \boldsymbol{\kappa}_{1}, \boldsymbol{\kappa}^{\prime}\right) d \boldsymbol{\kappa}_{1} \\
& +\sum_{l=1}^{j-1} \iint \widehat{\mathcal{T}}_{j-l}\left(\omega, z, \boldsymbol{\kappa}, \boldsymbol{\kappa}_{1}\right) \widehat{\mathcal{S}}_{\omega}^{+}\left(z, \boldsymbol{\kappa}_{1}, \boldsymbol{\kappa}_{2}\right) \widehat{\mathcal{R}}_{l}\left(\omega, z, \boldsymbol{\kappa}_{2}, \boldsymbol{\kappa}^{\prime}\right) d \boldsymbol{\kappa}_{1} d \boldsymbol{\kappa}_{2}
\end{aligned}
$$

for $j \geq 2$, with the initial conditions $\widehat{\mathcal{T}}_{1}\left(\omega, z=0, \boldsymbol{\kappa}, \boldsymbol{\kappa}^{\prime}\right)=\delta\left(\boldsymbol{\kappa}-\boldsymbol{\kappa}^{\prime}\right)$ and $\widehat{\mathcal{T}}_{j}\left(\omega, z=0, \boldsymbol{\kappa}, \boldsymbol{\kappa}^{\prime}\right)=0$ for all $j \geq 2$.

Note that the first-order term $\widehat{\mathcal{T}}_{1}$ is precisely the propagator of the paraxial wave equation. The higher-order terms $\widehat{\mathcal{T}}_{j}$ are corrections taking into account backscattering by the random medium.

\section{Statistical Analysis of the Reflected Wave}

\subsection{Scaled Regime}

Our goal is to show that the two-way paraxial wave equation is a simplified system that predicts accurately the statistics of the reflected wave in a special asymptotic regime. We consider the following scaled model for the bulk modulus and density

$$
\begin{aligned}
& \frac{1}{K^{\varepsilon}(z, \mathbf{x})}= \begin{cases}K_{0}^{-1} & \text { if } z \in(-\infty, 0) \cup(L, \infty), \\
K_{0}^{-1}\left(1+\varepsilon \nu\left(\frac{z}{\varepsilon^{2}}, \frac{\mathbf{x}}{\varepsilon}\right)\right) & \text { if } z \in(0, L),\end{cases} \\
& \rho^{\varepsilon}(z, \mathbf{x})=\rho_{0}
\end{aligned}
$$

where $\varepsilon$ is a small parameter and $\nu$ is a stationary zero-mean random process with the autocorrelation function

$$
C(z, \mathbf{x})=\mathbb{E}\left[\nu\left(z^{\prime}, \mathbf{x}^{\prime}\right) \nu\left(z^{\prime}+z, \mathbf{x}^{\prime}+\mathbf{x}\right)\right] .
$$

We assume that the incoming wave at $z=L$ has the scaled form

$$
p_{\text {inc }}^{\varepsilon}(t, \mathbf{x})=\frac{1}{2 \pi \varepsilon} \int \check{b}_{\text {inc }}\left(\omega, \frac{\mathbf{x}}{\varepsilon}\right) e^{-i \frac{\omega L}{c_{0} \varepsilon^{2}}} e^{-i \frac{\omega t}{\varepsilon^{2}}} d \omega .
$$

This means that the incoming wave has a carrier frequency of order $\varepsilon^{-2}$, a bandwidth of order one, and a transverse spatial extent of order $\varepsilon$. It has an amplitude of order $\varepsilon^{-1}$, which will ensure that the reflected wave is of order one (we are in a regime where backscattering is weak). This scaling is such that the 
Rayleigh distance of the incoming beam is of order one (it is proportional to the square of the input beam width divided by the carrier wavelength). Hence, the Rayleigh distance is of the same order as the size, $L$, of the random medium. Moreover, in the regime we consider, the correlation radius of the fluctuations of the random medium in the longitudinal direction is of the same order as the carrier wavelength (i.e., of order $\varepsilon^{2}$ ), while the correlation radius of the fluctuations of the random medium in the transverse direction is of the same order as the beam radius (i.e., of order $\varepsilon$ ). This resonant regime is therefore the one in which the wave is very sensitive to the fluctuations of the random medium.

We introduce the scaled right- and left-going mode amplitudes $\check{a}^{\varepsilon}$ and $\check{b}^{\varepsilon}$ :

$$
\begin{aligned}
& p^{\varepsilon}(t, z, \mathbf{x})=\frac{1}{2 \pi} \int\left(\check{a}^{\varepsilon}\left(\omega, z, \frac{\mathbf{x}}{\varepsilon}\right) e^{i \frac{\omega z}{c_{0} \varepsilon^{2}}}+\check{b}^{\varepsilon}\left(\omega, z, \frac{\mathbf{x}}{\varepsilon}\right) e^{-i \frac{\omega z}{c_{0} \varepsilon^{2}}}\right) e^{-i \frac{\omega t}{\varepsilon^{2}}} d \omega \\
& \frac{\partial p^{\varepsilon}}{\partial z}(t, z, \mathbf{x})=\frac{1}{2 \pi} \int \frac{i \omega}{c_{0} \varepsilon^{2}}\left(\check{a}^{\varepsilon}\left(\omega, z, \frac{\mathbf{x}}{\varepsilon}\right) e^{i \frac{\omega z}{c_{0} \varepsilon^{2}}}-\check{b}^{\varepsilon}\left(\omega, z, \frac{\mathbf{x}}{\varepsilon}\right) e^{-i \frac{\omega z}{c_{0} \varepsilon^{2}}}\right) e^{-i \frac{\omega t}{\varepsilon^{2}}} d \omega
\end{aligned}
$$

The equations for the iterative scheme for the mode amplitudes $\check{a}^{\varepsilon}(\omega, z, \mathbf{x})$ and $\check{b}^{\varepsilon}(\omega, z, \mathbf{x})$ are the ones given in (12-13), but with the $\varepsilon$-dependent operators

$$
\mathcal{L}_{\omega}^{\varepsilon}(z)=\frac{i \omega}{2 c_{0} \varepsilon} \nu\left(\frac{z}{\varepsilon^{2}}, \mathbf{x}\right)+\frac{i c_{0}}{2 \omega} \Delta_{\perp}, \quad \mathcal{S}_{\omega}^{ \pm, \varepsilon}(z)=e^{ \pm 2 i \frac{\omega z}{c_{0} \varepsilon^{2}}} \frac{i \omega}{2 c_{0} \varepsilon} \nu\left(\frac{z}{\varepsilon^{2}}, \mathbf{x}\right) .
$$

Note that the hypothesis of Lemma 3.2 is not fulfilled in this scaled regime, since the amplitude of the fluctuations operator $\mathcal{S}_{\omega}^{ \pm, \varepsilon}$ is of order $\varepsilon^{-1}$. Therefore, we cannot claim here that the scheme (12-13) converges strongly as $n \rightarrow \infty$. Rather, we will show that the scheme (12-13) gives very good predictions for the statistics of the reflected wave field.

\subsection{Asymptotic Analysis of the Reflection Kernel Process for the Two-way Paraxial Scheme}

In this subsection we study the statistics of the reflected wave as given by the two-way paraxial equation. In Subsection 5.3 we will compare these results with the behavior of the reflected wave predicted by the full wave equation [10]. The reader should keep in mind that our main goal is to prove that the two systems give the same statistics for the reflected wave in the scaled regime considered in this section.

The next proposition shows that it is possible to compute the cross moments of the reflection operator predicted by the first-order two-way paraxial equation in the limit $\varepsilon \rightarrow 0$. The proof is based on diffusion approximation theory and follows exactly the strategy used in [10] to obtain the equivalent system 
for the full wave equation. Indeed, the random components of the operators $\mathcal{L}_{\omega}^{\varepsilon}(z)$ and $\mathcal{S}_{\omega}^{ \pm, \varepsilon}(z)$ are centered (in the sense that their statistical means are zero) and vary rapidly at the scale $\varepsilon^{2}$. This scaled regime corresponds to a diffusion approximation regime in which the overall effect of the fluctuations can be modeled by a diffusion process of order one in the limit $\varepsilon \rightarrow 0$. The approach for identifying the law of the reflected wave field as predicted by the two-way parabolic approximation is based on identifying transport equations for the moments of specific products of reflection process kernels. Owing to the nonlinear coupling term in (25) we obtain below first a system that couples moments at different orders of the reflection kernel process evaluated at different wave vectors. To obtain a concise description for these moments we introduce below a multi-index notation for the wave vectors at which the kernels in the product are evaluated. We remark also that we evaluate the kernels at nearby frequencies since a quantity of main interest, the spectrum of the reflected wave field, derives from such an expression in this scaling regime with a relatively high carrier frequency, we shall discuss this in more detail below. Explicitly indicating the $\varepsilon$ dependence for the family of reflection operators we then have:

Proposition 5.1 Let us introduce the notation: If $\boldsymbol{\kappa}_{p}(j), \boldsymbol{\kappa}_{p}^{\prime}(j) \in \mathbb{R}^{d}, j=$ $1, \ldots, n_{\mathbf{p}}$, then the multi-vector $\mathbf{p}$ is the set

$$
\mathbf{p}=\left\{\left(\boldsymbol{\kappa}_{p}(j), \boldsymbol{\kappa}_{p}^{\prime}(j)\right)\right\}_{j=1}^{n_{\mathbf{p}}}
$$

where $n_{\mathbf{p}}$ stands for the number of pairs of vectors in $\mathbf{p}$.

We introduce the moments of products of $\widehat{\boldsymbol{\mathcal { R }}}^{\varepsilon,(1)}\left(\omega, z, \boldsymbol{\kappa}, \boldsymbol{\kappa}^{\prime}\right)$, the reflection process, at two nearby frequencies:

$$
\begin{aligned}
\mathcal{U}_{\mathbf{p}, \mathbf{q}}^{\varepsilon,(1)}(\omega, h, z)= \\
\quad \mathbb{E}\left[\prod_{j=1}^{n_{\mathbf{p}}} \widehat{\mathcal{R}}^{\varepsilon,(1)}\left(\omega+\frac{\varepsilon^{2} h}{2}, z, \boldsymbol{\kappa}_{p}(j), \boldsymbol{\kappa}_{p}^{\prime}(j)\right) \prod_{l=1}^{n_{\mathbf{q}}} \overline{\widehat{\mathcal{R}}^{\varepsilon,(1)}}\left(\omega-\frac{\varepsilon^{2} h}{2}, z, \boldsymbol{\kappa}_{q}(l), \boldsymbol{\kappa}_{q}^{\prime}(l)\right)\right],
\end{aligned}
$$

where $\mathbf{p}, \mathbf{q}$ are two multi-vectors of the form (33). We introduce the power spectral density of the fluctuations of the medium

$$
\widehat{C}(k, \boldsymbol{\kappa})=\int_{\mathbb{R}^{d}} \int_{-\infty}^{\infty} C(z, \mathbf{x}) e^{-i(k z+\boldsymbol{\kappa} \cdot \mathbf{x})} d z d \mathbf{x}
$$

The family of Fourier transforms

$$
W_{\mathbf{p}, \mathbf{q}}^{\varepsilon,(1)}(\omega, \tau, z)=\frac{1}{2 \pi} \int e^{-i h\left[\tau-\left(n_{\mathbf{p}}+n_{\mathbf{q}}\right) z / c_{0}\right]} \mathcal{U}_{\mathbf{p}, \mathbf{q}}^{\varepsilon,(1)}(\omega, h, z) d h
$$


converges as $\varepsilon \rightarrow 0$ to the solution $W_{\mathbf{p}, \mathbf{q}}^{(1)}$ of the system of transport equations

$$
\frac{\partial W_{\mathbf{p}, \mathbf{q}}^{(1)}}{\partial z}+\frac{n_{\mathbf{p}}+n_{\mathbf{q}}}{c_{0}} \frac{\partial W_{\mathbf{p}, \mathbf{q}}^{(1)}}{\partial \tau}=\frac{i c_{0}}{2 \omega} \Phi_{\mathbf{p}, \mathbf{q}} W_{\mathbf{p}, \mathbf{q}}^{(1)}+\frac{\omega^{2}}{4(2 \pi)^{d} c_{0}^{2}}\left(\mathcal{K} W^{(1)}+\mathcal{L} W^{(1)}\right)_{\mathbf{p}, \mathbf{q}},
$$

with the initial conditions $W_{\mathbf{p}, \mathbf{q}}^{(1)}(\omega, \tau, z=0)=\mathbf{1}_{0}\left(n_{\mathbf{p}}\right) \mathbf{1}_{0}\left(n_{\mathbf{q}}\right) \delta(\tau)$. Here we have defined

$$
\Phi_{\mathbf{p}, \mathbf{q}}=-\sum_{j=1}^{n_{\mathbf{p}}}\left(\left|\boldsymbol{\kappa}_{p}(j)\right|^{2}+\left|\boldsymbol{\kappa}_{p}^{\prime}(j)\right|^{2}\right)+\sum_{l=1}^{n_{\mathbf{q}}}\left(\left|\boldsymbol{\kappa}_{q}(l)\right|^{2}+\left|\boldsymbol{\kappa}_{q}^{\prime}(l)\right|^{2}\right),
$$

and the linear operators $\mathcal{K}$ and $\mathcal{L}$ are given by

$(\mathcal{K} W)_{\mathbf{p}, \mathbf{q}}=\sum_{j=1}^{n_{\mathbf{p}}} \sum_{l=1}^{n_{\mathbf{q}}} \widehat{C}\left(\frac{2 \omega}{c_{0}}, \boldsymbol{\kappa}_{p}(j)-\boldsymbol{\kappa}_{p}^{\prime}(j)\right) \delta\left(\boldsymbol{\kappa}_{p}(j)-\boldsymbol{\kappa}_{p}^{\prime}(j)-\boldsymbol{\kappa}_{q}(l)+\boldsymbol{\kappa}_{q}^{\prime}(l)\right) W_{\mathbf{p}|j, \mathbf{q}| l}$,

$$
\begin{aligned}
& (\mathcal{L} W)_{\mathbf{p}, \mathbf{q}}=\int d \boldsymbol{\kappa} \widehat{C}(0, \boldsymbol{\kappa})\left\{-\left(n_{\mathbf{p}}+n_{\mathbf{q}}\right) W_{\mathbf{p}, \mathbf{q}}\right. \\
& -\sum_{j=1}^{n_{\mathbf{p}}} W_{\mathbf{p} \mid\left\{j \mid\left(\boldsymbol{\kappa}_{p}(j)-\boldsymbol{\kappa}, \boldsymbol{\kappa}_{p}^{\prime}(j)-\boldsymbol{\kappa}\right)\right\}, \mathbf{q}}+\sum_{l=1}^{n_{\mathbf{q}}} W_{\mathbf{p}, \mathbf{q} \mid\left\{l \mid\left(\boldsymbol{\kappa}_{q}(l)-\boldsymbol{\kappa}, \boldsymbol{\kappa}_{q}^{\prime}(l)-\boldsymbol{\kappa}\right)\right.}
\end{aligned}
$$



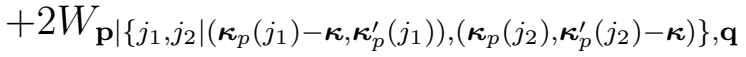

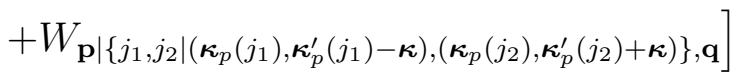

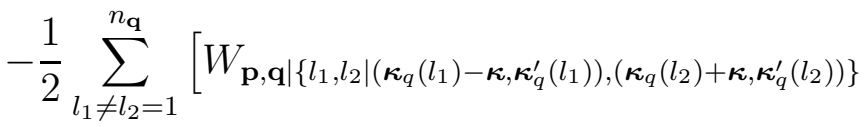

$$
\begin{aligned}
& +2 W_{\mathbf{p}, \mathbf{q} \mid\left\{l_{1}, l_{2} \mid\left(\boldsymbol{\kappa}_{q}\left(l_{1}\right)-\boldsymbol{\kappa}, \boldsymbol{\kappa}_{q}^{\prime}\left(l_{1}\right)\right),\left(\boldsymbol{\kappa}_{q}\left(l_{2}\right), \boldsymbol{\kappa}_{q}^{\prime}\left(l_{2}\right)-\boldsymbol{\kappa}\right)\right\}}
\end{aligned}
$$

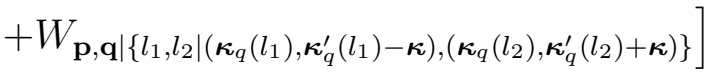

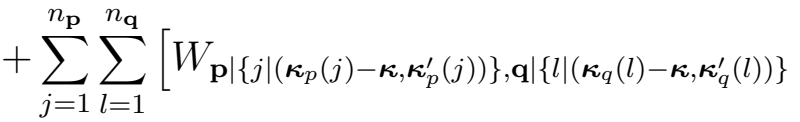

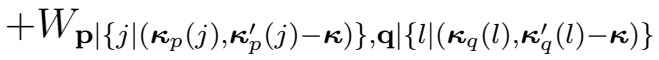

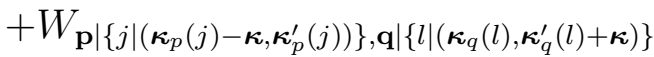

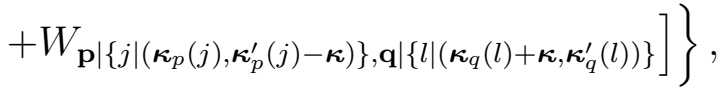

where we have used the notations:

$$
\begin{aligned}
& \mathbf{p} \mid j=\left\{\left(\boldsymbol{\kappa}_{p}\left(j^{\prime}\right), \boldsymbol{\kappa}_{p}^{\prime}\left(j^{\prime}\right)\right)\right\}_{j^{\prime}=1 \neq j}^{n_{\mathbf{p}}}, \\
& \mathbf{p} \mid\left\{j \mid\left(\boldsymbol{\kappa}_{1}, \boldsymbol{\kappa}_{2}\right)\right\}=\left\{\left(\boldsymbol{\kappa}_{p}\left(j^{\prime}\right), \boldsymbol{\kappa}_{p}^{\prime}\left(j^{\prime}\right)\right)\right\}_{j^{\prime}=1 \neq j}^{n_{\mathbf{p}}} \cup\left(\boldsymbol{\kappa}_{1}, \boldsymbol{\kappa}_{2}\right) .
\end{aligned}
$$


Note that $(\mathcal{L} W)_{\mathbf{p}, \mathbf{q}}$ involves only terms $W_{\mathbf{p}^{\prime}, \mathbf{q}^{\prime}}$ with $n_{\mathbf{p}^{\prime}}=n_{\mathbf{p}}$ and $n_{\mathbf{q}^{\prime}}=n_{\mathbf{q}}$, while $(\mathcal{K} W)_{\mathbf{p}, \mathbf{q}}$ involves only terms $W_{\mathbf{p}^{\prime}, \mathbf{q}^{\prime}}$ with $n_{\mathbf{p}^{\prime}}=n_{\mathbf{p}}-1$ and $n_{\mathbf{q}^{\prime}}=n_{\mathbf{q}}-1$. Therefore, for any integer $N$, the system for $\left(W_{\mathbf{p}, \mathbf{q}}^{(1)}\right)$ restricted to the multivectors $(\mathbf{p}, \mathbf{q})$ such that $n_{\mathbf{p}} \leq N$ and $n_{\mathbf{q}} \leq N$ is closed. This sub-system characterizes the $2 N$-th order statistics of the reflected wave. We will focus our attention on the second-order statistics in Subsection 5.5.

Proposition 5.1 describes the statistics of the reflected wave as predicted by the first-order two-way paraxial equation $(n=1$ in (12-13)). We would like now to describe the statistics of the reflected wave as predicted by the secondorder two-way paraxial equation $(n=2$ in (12-13)). We introduce therefore the moments in (34) with the second- rather than first-order reflection kernel processes and study its asymptotic behavior. The next proposition characterizes the statistics of the reflection operator predicted by the second-order two-way scheme.

Proposition 5.2 We introduce the moments of products of $\widehat{\mathcal{R}}^{\varepsilon,(2)}\left(\omega, z, \boldsymbol{\kappa}, \boldsymbol{\kappa}^{\prime}\right)$ at two nearby frequencies:

$$
\begin{aligned}
& \mathcal{U}_{\mathbf{p}, \mathbf{q}}^{\varepsilon,(2)}(\omega, h, z)= \\
& \mathbb{E}\left[\prod_{j=1}^{n_{\mathbf{p}}} \widehat{\mathcal{R}}^{\varepsilon,(2)}\left(\omega+\frac{\varepsilon^{2} h}{2}, z, \boldsymbol{\kappa}_{p}(j), \boldsymbol{\kappa}_{p}^{\prime}(j)\right) \prod_{l=1}^{n_{\mathrm{q}}} \overline{\widehat{\mathcal{R}}^{\varepsilon,(2)}}\left(\omega-\frac{\varepsilon^{2} h}{2}, z, \boldsymbol{\kappa}_{q}(l), \boldsymbol{\kappa}_{q}^{\prime}(l)\right)\right],
\end{aligned}
$$

where $\mathbf{p}, \mathbf{q}$ are two multi-vectors of the form (33). The family of Fourier transforms

$$
W_{\mathbf{p}, \mathbf{q}}^{\varepsilon,(2)}(\omega, \tau, z)=\frac{1}{2 \pi} \int e^{-i h\left[\tau-\left(n_{\mathbf{p}}+n_{\mathbf{q}}\right) z / c_{0}\right]} \mathcal{U}_{\mathbf{p}, \mathbf{q}}^{\varepsilon,(2)}(\omega, h, z) d h,
$$

converges as $\varepsilon \rightarrow 0$ :

$$
\lim _{\varepsilon \rightarrow 0} W_{\mathbf{p}, \mathbf{q}}^{\varepsilon,(2)}(\omega, \tau, z)=W_{\mathbf{p}, \mathbf{q}}^{(2)}(\omega, \tau, z):=\sum_{\tilde{\mathbf{p}} \subset \mathbf{p}, \tilde{\mathbf{q}} \subset \mathbf{q}} \widetilde{W}_{\mathbf{p} \backslash \tilde{\mathbf{p}}, \mathbf{q} \backslash \tilde{\mathbf{q}}, \tilde{\mathbf{p}}, \tilde{\mathbf{q}}}(\omega, \tau, z),
$$

where $\widetilde{W}_{\mathbf{p}, \mathbf{q}, \tilde{\mathbf{p}}, \tilde{\mathbf{q}}}$ is the solution of

$$
\begin{aligned}
& \frac{\partial \widetilde{W}_{\mathbf{p}, \mathbf{q}, \tilde{\mathbf{p}}, \tilde{\mathbf{q}}}}{\partial z}+\frac{n_{\mathbf{p}}+n_{\mathbf{q}}+n_{\tilde{\mathbf{p}}}+n_{\tilde{\mathbf{q}}}}{c_{0}} \frac{\partial \widetilde{W}_{\mathbf{p}, \mathbf{q}, \tilde{\mathbf{p}}, \tilde{\mathbf{q}}}}{\partial \tau}=\frac{i c_{0}}{2 \omega}\left[\Phi_{\mathbf{p}, \mathbf{q}}+\Phi_{\tilde{\mathbf{p}}, \tilde{\mathbf{q}}}\right] \widetilde{W}_{\mathbf{p}, \mathbf{q}, \tilde{\mathbf{p}}, \tilde{\mathbf{q}}} \\
& \quad+\frac{\omega^{2}}{4(2 \pi)^{d} c_{0}^{2}}(\widetilde{\mathcal{K}} \widetilde{W}+\widetilde{\mathcal{L}} \widetilde{W}+\widetilde{\mathcal{M}} \widetilde{W})_{\mathbf{p}, \mathbf{q}, \tilde{\mathbf{p}}, \tilde{\mathbf{q}}},
\end{aligned}
$$

with the initial conditions $\widetilde{W}_{\mathbf{p}, \mathbf{q}, \tilde{\mathbf{p}}, \tilde{\mathbf{q}}}(\omega, \tau, z=0)=\mathbf{1}_{0}\left(n_{\mathbf{p}}\right) \mathbf{1}_{0}\left(n_{\mathbf{q}}\right) \mathbf{1}_{0}\left(n_{\tilde{\mathbf{p}}}\right) \mathbf{1}_{0}\left(n_{\tilde{\mathbf{q}}}\right) \delta(\tau)$. The linear operator $\tilde{\mathcal{K}}$ is defined exactly as in (39) and acts only on $(\mathbf{p}, \mathbf{q})$, not $(\tilde{\mathbf{p}}, \tilde{\mathbf{q}})$. The linear operator $\widetilde{\mathcal{L}}$ is defined as in (40), however, here acts on both $(\mathbf{p}, \mathbf{q})$ and $(\tilde{\mathbf{p}}, \tilde{\mathbf{q}})$. The linear operator $\widetilde{\mathcal{M}}$ is defined by 


$$
\begin{aligned}
& (\widetilde{\mathcal{M}} \widetilde{W})_{\mathbf{p}, \mathbf{q}, \tilde{\mathbf{p}}, \tilde{\mathbf{q}}}=-\sum_{\tilde{j}=1}^{n_{\tilde{\mathbf{p}}}} \int \widehat{C}^{+}\left(\frac{2 \omega}{c_{0}}, \boldsymbol{\kappa}\right) d \boldsymbol{\kappa} \widetilde{W}_{\mathbf{p} \cup\left(\boldsymbol{\kappa}_{\tilde{p}}(\tilde{j}), \boldsymbol{\kappa}_{\tilde{p}}^{\prime}(\tilde{j})\right), \mathbf{q}, \tilde{\mathbf{p}} \mid \tilde{j}, \tilde{\mathbf{q}}} \\
& -\sum_{\tilde{l}=1}^{n_{\tilde{\mathbf{q}}}} \int \widehat{C}^{-}\left(\frac{2 \omega}{c_{0}}, \boldsymbol{\kappa}\right) d \boldsymbol{\kappa} \widetilde{W}_{\mathbf{p}, \mathbf{q} \cup\left(\boldsymbol{\kappa}_{\tilde{q}}(\tilde{l}), \boldsymbol{\kappa}_{\tilde{q}}^{\prime}(\tilde{l})\right), \tilde{\mathbf{p}}, \tilde{\mathbf{q}} \mid \tilde{l}} \\
& -\sum_{j=1}^{n_{\mathbf{p}}} \sum_{\tilde{j}=1}^{n_{\tilde{\mathbf{p}}}} \int \widehat{C}\left(\frac{2 \omega}{c_{0}}, \boldsymbol{\kappa}_{p}(j)-\boldsymbol{\kappa}_{p}^{\prime}(j)\right) \widetilde{W}_{\mathbf{p}\left|\left\{j \mid\left(\boldsymbol{\kappa}_{\tilde{p}}(\tilde{j}), \boldsymbol{\kappa}-\boldsymbol{\kappa}_{p}(j)\right),\left(\boldsymbol{\kappa}-\boldsymbol{\kappa}_{p}^{\prime}(j), \boldsymbol{\kappa}_{\tilde{p}}^{\prime}(\tilde{j})\right)\right\}, \mathbf{q}, \tilde{\mathbf{p}}\right| \tilde{j}, \tilde{\mathbf{q}}} d \boldsymbol{\kappa} \\
& -\sum_{l=1}^{n_{\mathbf{q}}} \sum_{\tilde{l}=1}^{n_{\tilde{\mathbf{q}}}} \int \widehat{C}\left(\frac{2 \omega}{c_{0}}, \boldsymbol{\kappa}_{q}(l)-\boldsymbol{\kappa}_{q}^{\prime}(l)\right) \widetilde{W}_{\mathbf{p}, \mathbf{q}\left|\left\{l \mid\left(\boldsymbol{\kappa}_{\tilde{q}}(\tilde{l}), \boldsymbol{\kappa}-\boldsymbol{\kappa}_{q}(l)\right),\left(\boldsymbol{\kappa}-\boldsymbol{\kappa}_{q}^{\prime}(l), \boldsymbol{\kappa}_{\tilde{q}}^{\prime}(\tilde{l})\right)\right\}, \tilde{\mathbf{p}}, \tilde{\mathbf{q}}\right| \tilde{l}} d \boldsymbol{\kappa} \\
& +\sum_{\tilde{j}=1}^{n_{\tilde{\mathbf{p}}}} \sum_{\tilde{l}=1}^{n_{\tilde{\mathbf{q}}}} \iiint d \boldsymbol{\kappa}_{1} d \boldsymbol{\kappa}_{2} d \boldsymbol{\kappa}_{3} \widehat{C}\left(\frac{2 \omega}{c_{0}}, \boldsymbol{\kappa}_{1}\right)
\end{aligned}
$$

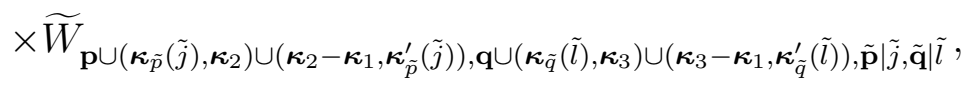

where

$$
\widehat{C}^{ \pm}(k, \boldsymbol{\kappa})=2 \int_{\mathbb{R}^{d}} \int_{0}^{\infty} C(z, \mathbf{x}) e^{ \pm i k z-i \boldsymbol{\kappa} \cdot \mathbf{x}} d z d \mathbf{x}
$$

Sketch of proof. From (23) we get

$$
\begin{aligned}
& \mathcal{U}_{\mathbf{p}, \mathbf{q}}^{\varepsilon,(2)}(\omega, h, z)=\sum_{\tilde{\mathbf{p}} \subset \mathbf{p}, \tilde{\mathbf{q}} \subset \mathbf{q}} \tilde{\mathcal{U}}_{\mathbf{p} \backslash \tilde{\mathbf{p}}, \mathbf{q} \backslash \tilde{\mathbf{q}}, \tilde{\mathbf{p}}, \tilde{\mathbf{q}}}^{\varepsilon}(\omega, h, z), \\
& \widetilde{\mathcal{U}}_{\mathbf{p}, \mathbf{q}, \tilde{\mathbf{p}}, \tilde{\mathbf{q}}}^{\varepsilon}(\omega, h, z)=\mathbb{E}\left[\prod_{j=1}^{n_{\mathbf{p}}} \widehat{\boldsymbol{\mathcal { R }}}_{1}^{\varepsilon}\left(\omega+\frac{\varepsilon^{2} h}{2}, z, \boldsymbol{\kappa}_{p}(j), \boldsymbol{\kappa}_{p}^{\prime}(j)\right) \prod_{l=1}^{n_{\mathbf{q}}} \overline{\widehat{\boldsymbol{\mathcal { R }}}_{1}^{\varepsilon}}\left(\omega-\frac{\varepsilon^{2} h}{2}, z, \boldsymbol{\kappa}_{q}(l), \boldsymbol{\kappa}_{q}^{\prime}(l)\right)\right. \\
& \left.\quad \times \prod_{\tilde{j}=1}^{n_{\tilde{\mathbf{p}}}} \widehat{\mathcal{R}}_{2}^{\varepsilon}\left(\omega+\frac{\varepsilon^{2} h}{2}, z, \boldsymbol{\kappa}_{\tilde{p}}(\tilde{j}), \boldsymbol{\kappa}_{\tilde{p}}^{\prime}(\tilde{j})\right) \prod_{\tilde{l}=1}^{n_{\tilde{\mathbf{q}}}} \overline{\widehat{\mathcal{R}}_{2}^{\varepsilon}}\left(\omega-\frac{\varepsilon^{2} h}{2}, z, \boldsymbol{\kappa}_{\tilde{q}}(\tilde{l}), \boldsymbol{\kappa}_{\tilde{q}}^{\prime}(\tilde{l})\right)\right],
\end{aligned}
$$

where $\widehat{\boldsymbol{\mathcal { R }}}_{1}^{\varepsilon}$ and $\widehat{\boldsymbol{\mathcal { R }}}_{2}^{\varepsilon}$ satisfy the $\varepsilon$-dependent versions of (24) and (25). The application of the diffusion approximation theory then gives the result.

Note that the last component of $(\widetilde{\mathcal{M}} \widetilde{W})_{\mathbf{p}, \mathbf{q}, \tilde{\mathbf{p}}, \tilde{\mathbf{q}}}$ involves terms $\widetilde{W}_{\mathbf{p}^{\prime}, \mathbf{q}^{\prime}, \tilde{\mathbf{p}}^{\prime}, \tilde{\mathbf{q}}^{\prime} \text { with }}$ $n_{\mathbf{p}^{\prime}}+n_{\tilde{\mathbf{p}}^{\prime}}=n_{\mathbf{p}}+n_{\tilde{\mathbf{p}}}+1$ and $n_{\mathbf{q}^{\prime}}+n_{\tilde{\mathbf{q}}^{\prime}}=n_{\mathbf{q}}+n_{\tilde{\mathbf{q}}}+1$. Therefore, it is not possible to isolate a closed sub-system to compute (for instance) the secondorder statistics of the reflected wave with this system.

\subsection{Asymptotic Analysis of the Reflection Kernel Process for the Full Wave Equation}

In [10] it is shown that the statistics of the reflection operator for the full wave equation is characterized by an infinite-dimensional system of transport 
equations. The limits of the Fourier transforms satisfy the system

$$
\begin{aligned}
\frac{\partial W_{\mathbf{p}, \mathbf{q}}}{\partial z}+\frac{n_{\mathbf{p}}+n_{\mathbf{q}}}{c_{0}} \frac{\partial W_{\mathbf{p}, \mathbf{q}}}{\partial \tau}= & \frac{i c_{0}}{2 \omega} \Phi_{\mathbf{p}, \mathbf{q}} W_{\mathbf{p}, \mathbf{q}}+\frac{\omega^{2}}{4(2 \pi)^{d} c_{0}^{2}}(\mathcal{K} W+\mathcal{L} W)_{\mathbf{p}, \mathbf{q}} \\
& +\frac{\omega^{2}}{4(2 \pi)^{d} c_{0}^{2}}(\mathcal{M} W+\mathcal{N} W)_{\mathbf{p}, \mathbf{q}}
\end{aligned}
$$

where the two additional linear operators $\mathcal{M}$ and $\mathcal{N}$ are defined by

$$
\begin{aligned}
& (\mathcal{M} W)_{\mathbf{p}, \mathbf{q}}=-\int n_{\mathbf{p}} \widehat{C}^{+}\left(\frac{2 \omega}{c_{0}}, \boldsymbol{\kappa}\right) d \boldsymbol{\kappa} W_{\mathbf{p}, \mathbf{q}}-\int n_{\mathbf{q}} \widehat{C}^{-}\left(\frac{2 \omega}{c_{0}}, \boldsymbol{\kappa}\right) d \boldsymbol{\kappa} W_{\mathbf{p}, \mathbf{q}} \\
& -\sum_{j_{1} \neq j_{2}=1}^{n_{\mathbf{p}}} \int \widehat{C}\left(\frac{2 \omega}{c_{0}}, \boldsymbol{\kappa}_{p}\left(j_{1}\right)-\boldsymbol{\kappa}_{p}^{\prime}\left(j_{1}\right)\right) W_{\left.\mathbf{p} \mid\left\{j_{1}, j_{2} \mid\left(\boldsymbol{\kappa}_{p}\left(j_{2}\right), \boldsymbol{\kappa}-\boldsymbol{\kappa}_{p}\left(j_{1}\right)\right),\left(\boldsymbol{\kappa}-\boldsymbol{\kappa}_{p}^{\prime}\left(j_{1}\right), \boldsymbol{\kappa}_{p}^{\prime}\left(j_{2}\right)\right)\right\}, \mathbf{q}\right)} d \boldsymbol{\kappa} \\
& -\sum_{l_{1} \neq l_{2}=1}^{n_{\mathbf{q}}} \int \widehat{C}\left(\frac{2 \omega}{c_{0}}, \boldsymbol{\kappa}_{q}\left(l_{1}\right)-\boldsymbol{\kappa}_{q}^{\prime}\left(l_{1}\right)\right) W_{\mathbf{p}, \mathbf{q} \mid\left\{l_{1}, l_{2} \mid\left(\boldsymbol{\kappa}_{q}\left(l_{2}\right), \boldsymbol{\kappa}-\boldsymbol{\kappa}_{q}\left(l_{1}\right)\right),\left(\boldsymbol{\kappa}-\boldsymbol{\kappa}_{q}^{\prime}\left(l_{1}\right), \boldsymbol{\kappa}_{q}^{\prime}\left(l_{2}\right)\right)\right\}} d \boldsymbol{\kappa},
\end{aligned}
$$

and

$$
\begin{aligned}
& (\mathcal{N} W)_{\mathbf{p}, \mathbf{q}}=\sum_{j=1}^{n_{\mathbf{p}}} \sum_{l=1}^{n_{\mathbf{q}}} \iiint \widehat{C}\left(\frac{2 \omega}{c_{0}}, \boldsymbol{\kappa}_{1}\right) \\
& \quad \times W_{\mathbf{p}\left|\left\{j \mid\left(\boldsymbol{\kappa}_{p}(j), \boldsymbol{\kappa}_{2}\right),\left(\boldsymbol{\kappa}_{2}-\boldsymbol{\kappa}_{1}, \boldsymbol{\kappa}_{p}^{\prime}(j)\right)\right\}, \mathbf{q}\right|\left\{l \mid\left(\boldsymbol{\kappa}_{q}(l), \boldsymbol{\kappa}_{3}\right),\left(\boldsymbol{\kappa}_{3}-\boldsymbol{\kappa}_{1}, \boldsymbol{\kappa}_{q}^{\prime}(l)\right)\right\}} d \boldsymbol{\kappa}_{1} d \boldsymbol{\kappa}_{2} d \boldsymbol{\kappa}_{3} .
\end{aligned}
$$

Note that $(\mathcal{M} W)_{\mathbf{p}, \mathbf{q}}$ involves only terms $W_{\mathbf{p}^{\prime}, \mathbf{q}^{\prime}}$ with $n_{\mathbf{p}^{\prime}}=n_{\mathbf{p}}$ and $n_{\mathbf{q}^{\prime}}=n_{\mathbf{q}}$, while $(\mathcal{N} W)_{\mathbf{p}, \mathbf{q}}$ involves only terms $W_{\mathbf{p}^{\prime}, \mathbf{q}^{\prime}}$ with $n_{\mathbf{p}^{\prime}}=n_{\mathbf{p}}+1$ and $n_{\mathbf{q}^{\prime}}=n_{\mathbf{q}}+1$. Therefore, again it is not possible to isolate a closed sub-system to compute (for instance) the $2 N$-th order statistics of the reflected wave, and the full system (46) is in general impossible to solve.

We next study the full system (46) in the so-called weak backscattering regime. This regime corresponds to the case in which

$$
\delta:=\sup _{\boldsymbol{\kappa} \in \mathbb{R}^{d}, \omega \in\left[\omega_{0}-B, \omega_{0}+B\right]} \frac{\widehat{C}\left(\frac{2 \omega}{c_{0}}, \boldsymbol{\kappa}\right)}{\widehat{C}(0, \mathbf{0})} \ll 1,
$$

where $\omega_{0}$ is the carrier frequency and $B$ is the bandwidth of the incoming beam. The dimensionless parameter $\delta$ is the ratio of the average intensity of random backscattering over the intensity of forward scattering. The regime $\delta \ll 1$ is interesting for many applications in geophysics and optical tomography, and it describes physically relevant phenomena, in particular interference effects such as the enhanced backscattering (or weak localization) phenomenon $[10]$. 
We renormalize the power spectral density of the fluctuations of the random medium and introduce the rescaled quantity $\widehat{C}_{0}$ :

$$
\widehat{C}\left(\frac{2 \omega}{c_{0}}, \boldsymbol{\kappa}\right)=\delta \widehat{C}_{0}\left(\frac{2 \omega}{c_{0}}, \boldsymbol{\kappa}\right), \quad \widehat{C}(0, \boldsymbol{\kappa})=\widehat{C}_{0}(0, \boldsymbol{\kappa}),
$$

which is such that $\widehat{C}_{0}\left(\frac{2 \omega}{c_{0}}, \boldsymbol{\kappa}\right)$ and $\widehat{C}_{0}(0, \boldsymbol{\kappa})$ are of the same order for $\omega$ in the spectrum of the incoming beam.

Proposition 5.3 The solution $W_{\mathbf{p}, \mathbf{q}}$ of the full system (46) is zero if $n_{\mathbf{p}} \neq n_{\mathbf{q}}$ and if $n_{\mathbf{p}}=n_{\mathbf{q}}$ it can then be expanded for any $n$ as

$$
W_{\mathbf{p}, \mathbf{q}}=\sum_{j=1}^{n} \delta^{n_{\mathbf{p}}+j-1} W_{j \mathbf{p , q}}+o\left(\delta^{n_{\mathbf{p}}+n-1}\right),
$$

where $W_{1}$ satisfies the system:

$$
\frac{\partial W_{1 \mathbf{p}, \mathbf{q}}}{\partial z}+\frac{n_{\mathbf{p}}+n_{\mathbf{q}}}{c_{0}} \frac{\partial W_{1 \mathbf{p}, \mathbf{q}}}{\partial \tau}=\frac{i c_{0}}{2 \omega} \Phi_{\mathbf{p}, \mathbf{q}} W_{1 \mathbf{p}, \mathbf{q}}+\frac{\omega^{2}}{4(2 \pi)^{d} c_{0}^{2}}\left(\mathcal{K}_{0} W_{1}+\mathcal{L}_{0} W_{1}\right)_{\mathbf{p}, \mathbf{q}},
$$

with the initial conditions $W_{1 \mathbf{p}, \mathbf{q}}(\omega, \tau, z=0)=\mathbf{1}_{0}\left(n_{\mathbf{p}}\right) \mathbf{1}_{0}\left(n_{\mathbf{q}}\right) \delta(\tau)$. Here $\mathcal{K}_{0}$ and $\mathcal{L}_{0}$ are the same operators as $\mathcal{K}$ and $\mathcal{L}$, but with $\widehat{C}_{0}$ instead of $\widehat{C} . W_{2}$ satisfies

$$
\begin{aligned}
\frac{\partial W_{2 \mathbf{p}, \mathbf{q}}}{\partial z}+\frac{n_{\mathbf{p}}+n_{\mathbf{q}}}{c_{0}} \frac{\partial W_{2 \mathbf{p}, \mathbf{q}}}{\partial \tau}= & \frac{i c_{0}}{2 \omega} \Phi_{\mathbf{p}, \mathbf{q}} W_{2 \mathbf{p}, \mathbf{q}}+\frac{\omega^{2}}{4(2 \pi)^{d} c_{0}^{2}}\left(\mathcal{K}_{0} W_{2}+\mathcal{L}_{0} W_{2}\right)_{\mathbf{p}, \mathbf{q}} \\
& +\frac{\omega^{2}}{4(2 \pi)^{d} c_{0}^{2}}\left(\mathcal{M}_{0} W_{1}\right)_{\mathbf{p}, \mathbf{q}}
\end{aligned}
$$

with the initial conditions $W_{2 \mathbf{p}, \mathbf{q}}(\omega, \tau, z=0)=0$. More generally the $W_{j}$ 's for $j \geq 3$ satisfy the systems:

$$
\begin{aligned}
\frac{\partial W_{j_{\mathbf{p}, \mathbf{q}}}}{\partial z}+\frac{n_{\mathbf{p}}+n_{\mathbf{q}}}{c_{0}} \frac{\partial W_{j_{\mathbf{p}, \mathbf{q}}}}{\partial \tau}= & \frac{i c_{0}}{2 \omega} \Phi_{\mathbf{p}, \mathbf{q}} W_{j} \mathbf{p , \mathbf { q }} \\
& +\frac{\omega^{2}}{4(2 \pi)^{d} c_{0}^{2}}\left(\mathcal{K}_{0} W_{j}+\mathcal{L}_{0} W_{j}\right)_{\mathbf{p}, \mathbf{q}} \\
& +\frac{\omega^{2}}{4(2 \pi)^{d} c_{0}^{2}}\left(\mathcal{M}_{0} W_{j-1}+\mathcal{N}_{0} W_{j-2}\right)_{\mathbf{p}, \mathbf{q}}
\end{aligned}
$$

with the initial conditions $W_{j_{\mathbf{p}, \mathbf{q}}}(\omega, \tau, z=0)=0$.

Note that, for any $n \geq 1$ and $N \geq 1$, the system for $\left(W_{j_{\mathbf{p}, \mathbf{q}}}\right)_{j=1, \ldots, n}$ restricted to the multi-vectors $(\mathbf{p}, \mathbf{q})$ such that $n_{\mathbf{p}} \leq N$ and $n_{\mathbf{q}} \leq N$ is closed. This sub-system characterizes the $2 N$-th order statistics of the reflected wave in the weak backscattering regime with a relative error of order $o\left(\delta^{n-1}\right)$.

We can revisit the asymptotic analysis of the statistics of the reflection operator predicted by the two-way paraxial scheme in the weak backscattering regime. 
Proposition 5.4 The solution $W_{\mathbf{p}, \mathbf{q}}^{(1)}$ obtained with the first-order two-way scheme (Proposition 5.1) is zero if $n_{\mathbf{p}} \neq n_{\mathbf{q}}$ and if $n_{\mathbf{p}}=n_{\mathbf{q}}$ it can be written for any $n$ as

$$
W_{\mathbf{p}, \mathbf{q}}^{(1)}=\delta^{n_{\mathbf{p}}} W_{1 \mathbf{p}, \mathbf{q}}
$$

where $W_{1}$ is the solution of (51).

The solution $W_{\mathbf{p}, \mathbf{q}}^{(2)}$ obtained with the second-order two-way scheme (Proposition 5.2) is zero if $n_{\mathbf{p}} \neq n_{\mathbf{q}}$ and if $n_{\mathbf{p}}=n_{\mathbf{q}}$ it can be expanded for any $n$ as

$$
W_{\mathbf{p}, \mathbf{q}}^{(2)}=\delta^{n_{\mathbf{p}}} W_{1 \mathbf{p}, \mathbf{q}}+\delta^{n_{\mathbf{p}}+1} W_{2 \mathbf{p}, \mathbf{q}}+o\left(\delta^{n_{\mathbf{p}}+1}\right),
$$

where $W_{2}$ is the solution of (52).

\subsection{Discussion}

We now notice the following important points which are the main results of this paper.

(1) By comparing (50) and (54), we find that in the weak backscattering regime, the first-order two-way paraxial equation reproduces correctly the statistics of the reflected wave to leading order.

(2) By comparing (50) and (55), we find that in the weak backscattering regime, the second-order two-way paraxial equation reproduces correctly the statistics of the reflected wave to second order.

(3) The theoretical question remains open whether the equivalence can be established at any order. Due to complicated algebra we were not able to write the full expansion of the moments of the reflection operator for the $n$-th order two-way paraxial equation, as we have done in (53) for the reflection operator of the full wave equation in the weak backscattering regime. However, it is natural to expect that the equivalence is maintained at any order.

\subsection{The Second-order Statistics}

A central quantity that characterizes the statistics of the backscattered wave field is the cross spectral density. We consider the cross spectral density obtained with the $n$-th order two-way paraxial scheme:

$$
\begin{aligned}
& W_{\left(\boldsymbol{\kappa}_{1}, \boldsymbol{\kappa}_{2}\right),\left(\boldsymbol{\kappa}_{3}, \boldsymbol{\kappa}_{4}\right)}^{(n)}(\omega, \tau, z)=\lim _{\varepsilon \rightarrow 0} \frac{1}{2 \pi} \int e^{-i h\left(\tau-2 z / c_{0}\right)} \\
& \times \mathbb{E}\left[\widehat{\boldsymbol{\mathcal { R }}}^{\varepsilon,(n)}\left(\omega+\frac{\varepsilon^{2} h}{2}, z, \boldsymbol{\kappa}_{1}, \boldsymbol{\kappa}_{2}\right) \widehat{\boldsymbol{\mathcal { R }}}^{\varepsilon,(n)}\left(\omega-\frac{\varepsilon^{2} h}{2}, z, \boldsymbol{\kappa}_{3}, \boldsymbol{\kappa}_{4}\right)\right] d h .
\end{aligned}
$$


This quantity describes the cross correlations of the reflected wave field

$$
\begin{array}{rl}
\lim _{\varepsilon \rightarrow 0} & \mathbb{E}\left[p_{\text {ref }}^{\varepsilon,(n)}(t, \varepsilon \mathbf{x}) p_{\text {ref }}^{\varepsilon,(n)}\left(t+\varepsilon^{2} s, \varepsilon \mathbf{x}^{\prime}\right)\right]=\frac{1}{(2 \pi)^{2 d+1}} \int \cdots \int W_{\left(\boldsymbol{\kappa}_{1}, \boldsymbol{\kappa}_{2}\right),\left(\boldsymbol{\kappa}_{3}, \boldsymbol{\kappa}_{4}\right)}^{(n)}(\omega, t, L) \\
& \times e^{i \boldsymbol{\kappa}_{1} \cdot \mathbf{x}-i \boldsymbol{\kappa}_{3} \cdot \mathbf{x}^{\prime}} e^{i \omega s} \hat{b}_{\text {inc }}\left(\omega, \boldsymbol{\kappa}_{2}\right) \overline{\hat{b}_{\text {inc }}}\left(\omega, \boldsymbol{\kappa}_{4}\right) d \omega d \boldsymbol{\kappa}_{1} d \boldsymbol{\kappa}_{2} d \boldsymbol{\kappa}_{3} d \boldsymbol{\kappa}_{4}
\end{array}
$$

where $p_{\text {ref }}^{\varepsilon,(n)}$ is the reflected field obtained by the $n$-th-order two-way paraxial equation:

$$
\begin{aligned}
p_{\text {ref }}^{\varepsilon,(n)}(t, \varepsilon \mathbf{x}) & =\frac{1}{2 \pi} \int \hat{a}_{n}^{\varepsilon}(\omega, L, \mathbf{x}) e^{i \frac{\omega L}{c_{0} \varepsilon^{2}}} e^{-i \frac{\omega t}{\varepsilon^{2}}} d \omega \\
& =\frac{1}{(2 \pi)^{d+1} \varepsilon} \iiint \widehat{\mathcal{R}}^{\varepsilon,(n)}\left(\omega, L, \boldsymbol{\kappa}, \boldsymbol{\kappa}^{\prime}\right) \hat{b}_{\mathrm{inc}}\left(\omega, \boldsymbol{\kappa}^{\prime}\right) e^{i \boldsymbol{\kappa} \cdot \mathbf{x}} d \boldsymbol{\kappa}^{\prime} d \boldsymbol{\kappa} e^{i \frac{\omega L}{c_{0} \varepsilon^{2}}} e^{-i \frac{\omega t}{\varepsilon^{2}}} d \omega
\end{aligned}
$$

As noted in Subsection 5.2, the cross spectral density for the first-order scheme can be characterized by a closed system of transport equations. We can write this closed system in a simple form, as shown in the next proposition.

Proposition 5.5 The cross spectral density $W_{\left(\boldsymbol{\kappa}_{1}, \boldsymbol{\kappa}_{2}\right),\left(\boldsymbol{\kappa}_{3}, \boldsymbol{\kappa}_{4}\right)}^{(1)}(\omega, \tau, z)$ defined by (56) for the first-order scheme has the form

$$
W_{\left(\boldsymbol{\kappa}_{1}, \boldsymbol{\kappa}_{2}\right),\left(\boldsymbol{\kappa}_{3}, \boldsymbol{\kappa}_{4}\right)}^{(1)}(\omega, \tau, z)=V_{\boldsymbol{\kappa}_{2}-\boldsymbol{\kappa}_{4}, \boldsymbol{\kappa}_{1}+\boldsymbol{\kappa}_{4}, \boldsymbol{\kappa}_{1}-\boldsymbol{\kappa}_{2}}(\omega, \tau, z) \delta\left(\boldsymbol{\kappa}_{1}-\boldsymbol{\kappa}_{2}-\boldsymbol{\kappa}_{3}+\boldsymbol{\kappa}_{4}\right),
$$

where $V_{\boldsymbol{\kappa}_{u}, \boldsymbol{\kappa}_{v}, \boldsymbol{\kappa}_{w}}(\omega, \tau, z)$ is the solution of the system of transport equations

$$
\begin{gathered}
\frac{\partial V_{\boldsymbol{\kappa}_{u}, \boldsymbol{\kappa}_{v}, \boldsymbol{\kappa}_{w}}}{\partial z}+\frac{2}{c_{0}} \frac{\partial V_{\boldsymbol{\kappa}_{u}, \boldsymbol{\kappa}_{v}, \boldsymbol{\kappa}_{w}}}{\partial \tau}=\frac{\omega^{2}}{4(2 \pi)^{d} c_{0}^{2}} \widehat{C}\left(\frac{2 \omega}{c_{0}}, \boldsymbol{\kappa}_{w}\right) \delta(\tau)-\frac{i c_{0}}{\omega} \boldsymbol{\kappa}_{u} \cdot \boldsymbol{\kappa}_{v} V_{\boldsymbol{\kappa}_{u}, \boldsymbol{\kappa}_{v}, \boldsymbol{\kappa}_{w}} \\
\quad+\frac{\omega^{2}}{4(2 \pi)^{d} c_{0}^{2}} \int \widehat{C}(0, \boldsymbol{\kappa})\left\{V_{\boldsymbol{\kappa}_{u}, \boldsymbol{\kappa}_{v}-\boldsymbol{\kappa}, \boldsymbol{\kappa}_{w}-\boldsymbol{\kappa}}+V_{\boldsymbol{\kappa}_{u}, \boldsymbol{\kappa}_{v}-\boldsymbol{\kappa}, \boldsymbol{\kappa}_{w}+\boldsymbol{\kappa}}+V_{\boldsymbol{\kappa}_{u}-\boldsymbol{\kappa}, \boldsymbol{\kappa}_{v}, \boldsymbol{\kappa}_{w}-\boldsymbol{\kappa}}\right. \\
\left.\quad+V_{\boldsymbol{\kappa}_{u}-\boldsymbol{\kappa}, \boldsymbol{\kappa}_{v}, \boldsymbol{\kappa}_{w}+\boldsymbol{\kappa}}-V_{\boldsymbol{\kappa}_{u}-\boldsymbol{\kappa}, \boldsymbol{\kappa}_{v}-\boldsymbol{\kappa}, \boldsymbol{\kappa}_{w}}-V_{\boldsymbol{\kappa}_{u}+\boldsymbol{\kappa}, \boldsymbol{\kappa}_{v}-\boldsymbol{\kappa}, \boldsymbol{\kappa}_{w}}-2 V_{\boldsymbol{\kappa}_{u}, \boldsymbol{\kappa}_{v}, \boldsymbol{\kappa}_{w}}\right\} d \boldsymbol{\kappa}
\end{gathered}
$$

starting from $V_{\boldsymbol{\kappa}_{u}, \boldsymbol{\kappa}_{v}, \boldsymbol{\kappa}_{w}}(\omega, \tau, z=0)=0$.

It follows from the results of Subsection 5.3 that the spectral density determined by the system (59) is the spectral density obtained from the full wave equation in the weak backscattering regime to leading order.

Proposition 5.6 The cross spectral density $W_{\left(\boldsymbol{\kappa}_{1}, \boldsymbol{\kappa}_{2}\right),\left(\boldsymbol{\kappa}_{3}, \boldsymbol{\kappa}_{4}\right)}^{(2)}(\omega, \tau, z)$ defined by (56) for the second-order scheme has the form

$$
W_{\left(\boldsymbol{\kappa}_{1}, \boldsymbol{\kappa}_{2}\right),\left(\boldsymbol{\kappa}_{3}, \boldsymbol{\kappa}_{4}\right)}^{(2)}(\omega, \tau, z)=W_{\left(\boldsymbol{\kappa}_{1}, \boldsymbol{\kappa}_{2}\right),\left(\boldsymbol{\kappa}_{3}, \boldsymbol{\kappa}_{4}\right)}^{(1)}(\omega, \tau, z) \exp \left(-\frac{\omega^{2} z}{2 c_{0}^{2}} \check{C}\left(\frac{2 \omega}{c_{0}}, 0\right)\right) .
$$


It follows from the results of Subsection 5.3 that the spectral density (60) is the spectral density obtained from the full wave equation in the weak backscattering regime to second-order.

Proof. Using (52) and

$$
\left[\mathcal{M}_{0} W_{1}\right]_{\left(\boldsymbol{\kappa}_{1}, \boldsymbol{\kappa}_{2}\right),\left(\boldsymbol{\kappa}_{3}, \boldsymbol{\kappa}_{4}\right)}=-2(2 \pi)^{d} \check{C}\left(\frac{2 \omega}{c_{0}}, 0\right) W_{1\left(\boldsymbol{\kappa}_{1}, \boldsymbol{\kappa}_{2}\right),\left(\boldsymbol{\kappa}_{3}, \boldsymbol{\kappa}_{4}\right)},
$$

we find by Duhamel's principle that the second-order correction is

$$
W_{2\left(\boldsymbol{\kappa}_{1}, \boldsymbol{\kappa}_{2}\right),\left(\boldsymbol{\kappa}_{3}, \boldsymbol{\kappa}_{4}\right)}(\omega, \tau, z)=-\frac{\omega^{2} z}{2 c_{0}^{2}} \check{C}_{0}\left(\frac{2 \omega}{c_{0}}, 0\right) W_{1\left(\boldsymbol{\kappa}_{1}, \boldsymbol{\kappa}_{2}\right),\left(\boldsymbol{\kappa}_{3}, \boldsymbol{\kappa}_{4}\right)}(\omega, \tau, z) .
$$

Therefore, using (55), we find

$$
W_{\left(\boldsymbol{\kappa}_{1}, \boldsymbol{\kappa}_{2}\right),\left(\boldsymbol{\kappa}_{3}, \boldsymbol{\kappa}_{4}\right)}^{(2)}(\omega, \tau, z)=\delta\left[1-\delta \frac{\omega^{2} z}{2 c_{0}^{2}} \check{C}_{0}\left(\frac{2 \omega}{c_{0}}, 0\right)\right] W_{1\left(\boldsymbol{\kappa}_{1}, \boldsymbol{\kappa}_{2}\right),\left(\boldsymbol{\kappa}_{3}, \boldsymbol{\kappa}_{4}\right)}(\omega, \tau, z),
$$

which is equivalent to second-order in $\delta$ to $(60)$.

As a first application, the mean reflected power defined by

$$
I^{\varepsilon}(t)=\int \mathbb{E}\left[p_{\mathrm{ref}}^{\varepsilon}(t, \mathbf{x})^{2}\right] d \mathbf{x}
$$

has the limit $I(t)$ as $\varepsilon \rightarrow 0$, with

$$
I(t)= \begin{cases}I_{0} & \text { if } t \in\left[0,2 L / c_{0}\right] \\ 0 & \text { otherwise }\end{cases}
$$

Here

$$
\begin{aligned}
I_{0} & =\frac{1}{(2 \pi)^{d+1}} \int \frac{\omega^{2}}{8 c_{0}} \check{C}\left(\frac{2 \omega}{c_{0}}, \mathbf{0}\right) \exp \left(-\frac{\omega^{2} L}{2 c_{0}^{2}} \check{C}\left(\frac{2 \omega}{c_{0}}, 0\right)\right)\left[\int\left|\hat{b}_{\mathrm{inc}}\left(\omega, \boldsymbol{\kappa}^{\prime}\right)\right|^{2} d \boldsymbol{\kappa}^{\prime}\right] d \omega \\
& \simeq \frac{\omega_{0}^{2}}{8 c_{0}} \check{C}\left(\frac{2 \omega_{0}}{c_{0}}, \mathbf{0}\right) \exp \left(-\frac{\omega_{0}^{2} L}{2 c_{0}^{2}} \check{C}\left(\frac{2 \omega_{0}}{c_{0}}, 0\right)\right)\left[\iint\left|b_{\text {inc }}(s, \mathbf{x})\right|^{2} d \mathbf{x} d s\right],
\end{aligned}
$$

where the second approximation is valid when the bandwidth of the incoming beam is small. See [10] regarding details of the calculation.

As a second application, the beam width $R^{\varepsilon}(t)$ defined by

$$
R^{\varepsilon 2}(t)=\frac{\int|\mathbf{x}|^{2} \mathbb{E}\left[p_{\text {ref }}^{\varepsilon}(t, \mathbf{x})^{2}\right] d \mathbf{x}}{\int \mathbb{E}\left[p_{\text {ref }}^{\varepsilon}(t, \mathbf{x})^{2}\right] d \mathbf{x}}
$$


has a limit $R(t)$ as $\varepsilon \rightarrow 0$, which is described in [10]. Let us denote by $l_{x}$ the transverse correlation radius of the medium and assume that $\check{C}(k, \mathbf{x})$ is twice differentiable at $\mathbf{x}=\mathbf{0}$. In the Fraunhofer regime $c_{0} L /\left(\omega_{0} l_{x}^{2}\right) \gg 1$ we obtain the approximate expression

$$
R^{2}(t)=R_{0}^{2}+\frac{c_{0}^{2}}{\omega_{0}^{2}}\left(4 K_{0}^{2}-\frac{\Delta_{\mathbf{x}} \check{C}\left(\frac{2 \omega_{0}}{c_{0}}, \mathbf{0}\right)}{\check{C}\left(\frac{2 \omega_{0}}{c_{0}}, \mathbf{0}\right)}\right)\left(\frac{c_{0} t}{2}\right)^{2}-\frac{2}{3} \Delta_{\mathbf{x}} \check{C}(0, \mathbf{0})\left(\frac{c_{0} t}{2}\right)^{3}
$$

where $R_{0}$ (resp. $K_{0}$ ) is the rms width (resp. spectral width) of the incoming beam defined by

$$
R_{0}^{2}=\frac{\iint|\mathbf{x}|^{2}\left|\check{b}_{\text {inc }}(\omega, \mathbf{x})\right|^{2} d \mathbf{x} d \omega}{\iint\left|\check{b}_{\text {inc }}(\omega, \mathbf{x})\right|^{2} d \mathbf{x} d \omega}, \quad K_{0}^{2}=\frac{\iint|\boldsymbol{\kappa}|^{2}\left|\hat{b}_{\mathrm{inc}}(\omega, \boldsymbol{\kappa})\right|^{2} d \boldsymbol{\kappa} d \omega}{\iint\left|\hat{b}_{\mathrm{inc}}(\omega, \boldsymbol{\kappa})\right|^{2} d \boldsymbol{\kappa} d \omega}
$$

\section{Simulations}

Comparisons between the Helmholtz equation and the two-way paraxial equations are given in $[12,13]$. In this section we will illustrate the main result of this paper, that the predictions of the numerical scheme for the statistics of the wave backscattered by a random medium are in agreement with the already known theoretical results.

We use a split-step Fourier method for solving the $1+1$-dimensional random paraxial wave equations (while a finite-difference scheme was used in $[12,13]$ ). In the numerical setup the size of the slab is $L=400$ and the background velocity is $c_{0}=1$. The random process $\nu(z, x)$ has the form

$$
\nu(z, x)=\sum_{j=0}^{\infty} \mathbf{1}_{\left[L_{j}, L_{j+1}\right)}(z) \nu_{j}(x)
$$

where $L_{0}=0, L_{j}=\sum_{i=1}^{j} l_{i} ; l_{i}$ are independent and identically distributed random variables with exponential distribution and mean $l_{z}=4 ; \nu_{j}(x)$ are independent and identically distributed Gaussian processes (in $x$ ) with Gaussian autocorrelation function, standard deviation $\sigma$ (with $\sigma=0.01$ or $\sigma=0.025$ ), and transverse correlation length $l_{x}=16$. The power spectral density of the random fluctuations is

$$
\widehat{C}(2 k, \kappa)=2 \sqrt{\pi} \sigma^{2} l_{x} l_{z} \frac{1}{1+4 k^{2} l_{z}^{2}} \exp \left(-\frac{\kappa^{2} l_{x}^{2}}{4}\right) .
$$

With $k_{0}=1$ and $l_{z}=4$, we have $\max _{\kappa} \widehat{C}\left(2 k_{0}, \kappa\right) / \widehat{C}(0,0) \simeq 0.016$ which shows that we are indeed in the weak backscattering regime. The incoming beam 
has a Gaussian shape in space with radius $r_{0}=32$ and a sinc shape in time with a central frequency $\omega_{0}=1$ and a bandwidth $B=0.1$ :

$$
b_{\text {inc }}(t, x)=\frac{1}{2} \operatorname{sinc}(B t) e^{-i \omega_{0} t} \exp \left(-\frac{x^{2}}{2 r_{0}^{2}}\right)+c c .
$$

Under these conditions, the mean reflected power is given by (61) with

$$
I_{0}=\frac{\omega_{0}^{2} \pi^{3 / 2} \sigma^{2} r_{0} l_{z}}{8 B c_{0}} \frac{1}{1+4 \omega_{0}^{2} l_{z}^{2} / c_{0}^{2}},
$$

and the beam width is approximately given by

$$
R^{2}(t)=\frac{r_{0}^{2}}{2}+\frac{c_{0}^{2}}{\omega_{0}^{2}}\left(\frac{1}{2 r_{0}^{2}}+\frac{1}{2 l_{x}^{2}}\right) c_{0}^{2} t^{2}+\frac{\sigma^{2} l_{z}}{3 l_{x}^{2}} c_{0}^{3} t^{3}
$$

In Figure 1 we compare these theoretical formulas with the numerical results given by the first-order two-way paraxial equation. The numerical average values are obtained from a set of 1000 simulations.

\section{Conclusion}

We have shown that the two-way paraxial equation predicts the statistical behavior of the wave reflected by a random medium with a computational cost equivalent to the standard paraxial wave equation, but with an accuracy equivalent to the one of the full wave equation, provided that:

1) The beam width must be larger than the carrier wavelength, corresponding to a paraxial regime for the transmitted wave.

2) The transverse correlation radius of the fluctuations of the medium parameters must be larger than the carrier wavelength, corresponding to a paraxial regime for the backscattered wave.

We have proved that the relative error between the statistics of the reflected wave field given by the first-order (resp. second-order) two-way paraxial equation and the one given by the full wave equation is of order $o(1)(\operatorname{resp} . o(\delta))$, where $\delta$ is the ratio of the average intensity of random backscattering over the one of forward scattering. We have also given heuristic arguments to support the conjecture that the relative error for the $n$-th order two-way paraxial equation should be of order $o\left(\delta^{n-1}\right)$.

We remark that a similar result should hold true for the transmitted wave. We have seen that the first-order two-way paraxial equation is equivalent to the standard paraxial wave equation for the transmitted wave and that the higherorder corrections describe the corrections due to random backscattering. By a strategy as the one presented in this paper, that is, by comparing the system 
a)

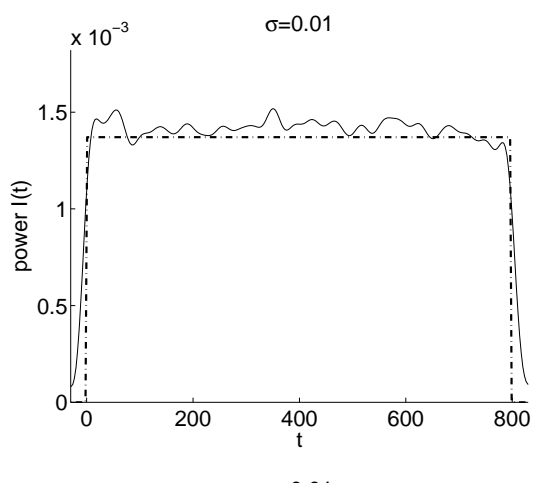

c)

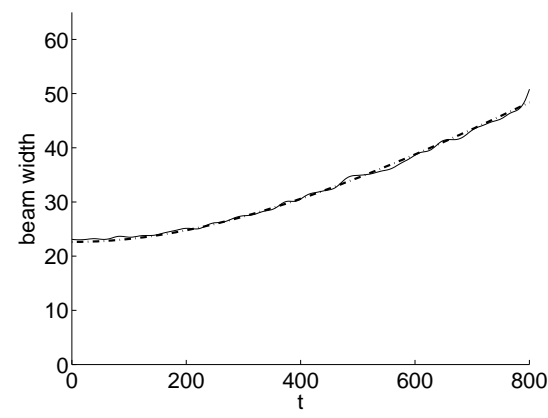

$\sigma=0.01$

e)

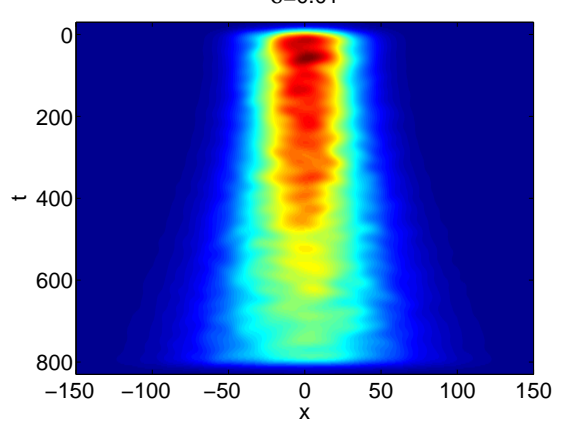

b)

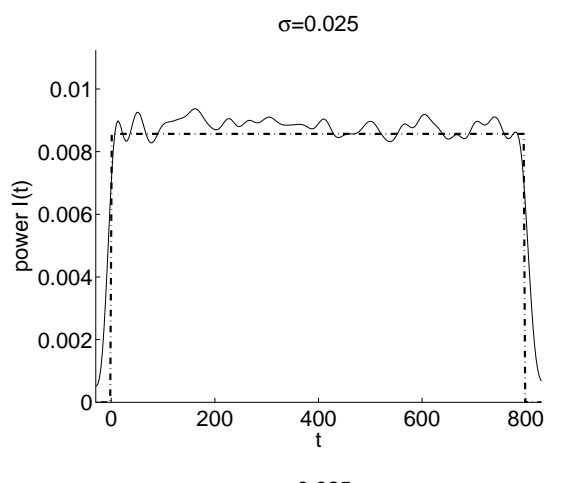

d)
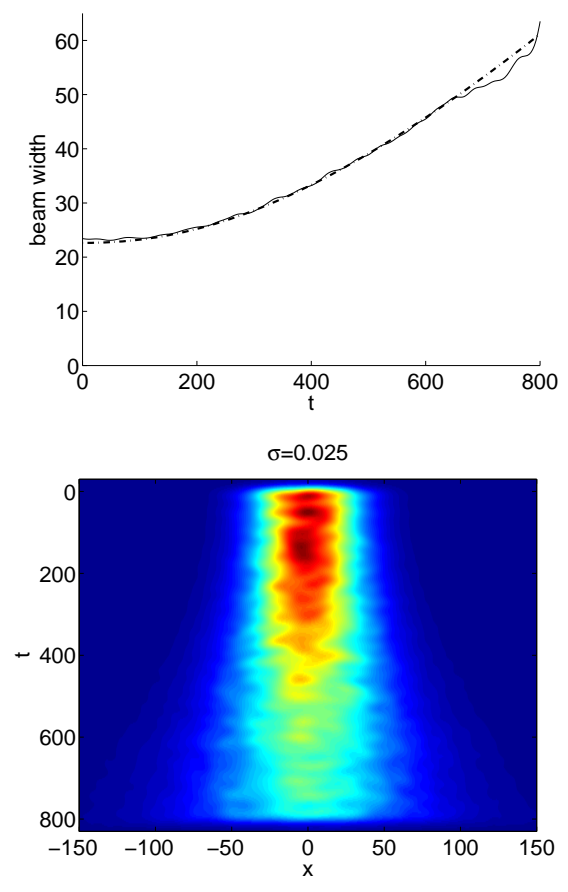

Fig. 1. The reflected powers, beam widths, and spatial profiles of the reflected beam as a function of time for $\sigma=0.01(\mathrm{a}, \mathrm{c}, \mathrm{e})$ and $\sigma=0.025(\mathrm{~b}, \mathrm{~d}, \mathrm{f})$. The thin solid lines are the numerical results and the thick dashed lines the theoretical formulas (61), (63) and (64). In the case $\sigma=0.01$ random forward scattering is rather weak. In the case $\sigma=0.025$ random forward scattering is rather strong.

of moments for the transmission operator given by the iterative scheme and the one given by the full system [10], one should be able to show that the error in the statistics of the transmitted wave field given by the $n$-th order two-way paraxial equation is of order $o\left(\delta^{n-1}\right)$.

The framework we have set forth, with an iterative implementation of a twoway paraxial system, is able to simulate realizations of random transmitted and reflected wave fields with the correct law in the asymptotic regime we have considered here. To the best of our knowledge there are no other numerical method that can accomplish this with a comparable computational cost. Therefore, we expect the scheme to be useful in the context of analyzing 
for instance imaging methods in regimes with relatively small scale medium features and wavelength and we refer to [11] for an example.

\section{Acknowledgements}

This work was supported by ONR grant N00014-02-1-0089 and DARPA grant N00014-05-1-0442. K. Sølna was supported by NSF grant DMS0307011 and the Sloan Foundation.

\section{A An Elementary Proof of Convergence of the Iterative Scheme}

The integral formulation of the problem is more convenient for the analysis. Let us introduce the unitary flow $U_{\omega}\left(z, z^{\prime}\right)$ from $L^{2}\left(\mathbb{R}^{d}\right)$ onto $L^{2}\left(\mathbb{R}^{d}\right)$ (and also from $H^{k}\left(\mathbb{R}^{d}\right)$ onto $\left.H^{k}\left(\mathbb{R}^{d}\right)\right)$ that is the fundamental solution of

$$
\frac{\partial U_{\omega}}{\partial z}\left(z, z^{\prime}\right)=\frac{i c_{0}}{2 \omega} \Delta_{\perp} U_{\omega}\left(z, z^{\prime}\right), \quad U_{\omega}\left(z^{\prime}, z^{\prime}\right)=I_{d}
$$

Its kernel is

$$
U_{\omega}\left(z, z^{\prime} ; \mathbf{x}, \mathbf{x}^{\prime}\right)=\left(\frac{i 2 \pi c_{0}\left(z-z^{\prime}\right)}{\omega}\right)^{d / 2} \exp \left[i \frac{\omega\left|\mathbf{x}-\mathbf{x}^{\prime}\right|^{2}}{2 c_{0}\left(z-z^{\prime}\right)}\right]
$$

with the convention $U_{\omega}\left(z^{\prime}, z^{\prime} ; \mathbf{x}, \mathbf{x}^{\prime}\right)=\delta\left(\mathbf{x}-\mathbf{x}^{\prime}\right)$. The integral formulation of the scheme is

$$
\begin{aligned}
\check{b}_{n}(\omega, z)= & U_{\omega}(z, L) \check{b}_{\mathrm{inc}}(\omega) \\
& +\frac{i \omega}{2 c_{0}} \int_{z}^{L} U_{\omega}\left(z, z^{\prime}\right) \nu\left(z^{\prime}\right)\left(\check{b}_{n}\left(\omega, z^{\prime}\right)+e^{-2 i \frac{\omega}{c_{0}} z^{\prime}} \check{a}_{n-1}\left(\omega, z^{\prime}\right)\right) d z^{\prime} \\
\check{a}_{n}(\omega, z)= & \frac{i \omega}{2 c_{0}} \int_{0}^{z} U_{\omega}\left(z, z^{\prime}\right) \nu\left(z^{\prime}\right)\left(\check{a}_{n}\left(\omega, z^{\prime}\right)+e^{2 i \frac{\omega}{c_{0}} z^{\prime}} \check{b}_{n}\left(\omega, z^{\prime}\right)\right) d z^{\prime}
\end{aligned}
$$

where we interpret $\nu(z)$ as a multiplication operator. We can now give the proof of Lemma 3.2.

Proof. Let us denote

$$
\delta \check{a}_{n}=\check{a}_{n+1}-\check{a}_{n}, n \geq 0, \quad \delta \check{b}_{n}=\check{b}_{n+1}-\check{b}_{n}, n \geq 1
$$


They satisfy for any $n \geq 1$

$$
\frac{\partial \delta \check{b}_{n}}{\partial z}=-\mathcal{L}_{\omega} \delta \check{b}_{n}-\mathcal{S}_{\omega}^{+} \delta \check{a}_{n-1}, \quad \frac{\partial \delta \check{a}_{n}}{\partial z}=\mathcal{L}_{\omega} \delta \check{a}_{n}+\mathcal{S}_{\omega}^{-} \delta \check{b}_{n},
$$

with the boundary conditions $\delta \check{a}_{n}(\omega, z=0, \mathbf{x})=0$ and $\delta \check{b}_{n}(\omega, z=L, \mathbf{x})=0$.

We have

$$
\begin{aligned}
& \frac{d\left\|\delta \check{b}_{n}(\omega, z)\right\|_{2}^{2}}{d z}=\Re\left(\frac{i \omega}{c_{0}} e^{-2 i \frac{\omega}{c_{0}} z} \int \nu(z, \mathbf{x}) \overline{\delta \check{b}_{n}}(\omega, z, \mathbf{x}) \delta \check{a}_{n-1}(\omega, z, \mathbf{x}) d \mathbf{x}\right), \\
& \frac{d\left\|\delta \check{a}_{n}(\omega, z)\right\|_{2}^{2}}{d z}=\Re\left(-\frac{i \omega}{c_{0}} e^{2 i \frac{\omega}{c_{0}} z} \int \nu(z, \mathbf{x}) \overline{\delta \check{a}_{n}}(\omega, z, \mathbf{x}) \delta \check{b}_{n}(\omega, z, \mathbf{x}) d \mathbf{x}\right) .
\end{aligned}
$$

Using Cauchy-Schwarz inequality, we find

$$
\left|\frac{d\left\|\delta \check{b}_{n}\right\|_{2}}{d z}\right| \leq C\left\|\delta \check{a}_{n-1}\right\|_{2}, \quad\left|\frac{d\left\|\delta \check{a}_{n}\right\|_{2}}{d z}\right| \leq C\left\|\delta \check{b}_{n}\right\|_{2},
$$

where $C=\left(\omega\|\nu\|_{\infty}\right) / c_{0}$. By integrating we obtain

$$
\left\|\delta \check{a}_{n}(z)\right\|_{2} \leq C^{2} \int_{0}^{L} d z^{\prime}\left(z \wedge z^{\prime}\right)\left\|\delta \check{a}_{n-1}\left(z^{\prime}\right)\right\|_{2} d z^{\prime} \leq \frac{3 C^{2} L^{2}}{4} \sup _{z^{\prime} \in[0, L]}\left\|\delta \check{a}_{n-1}\left(z^{\prime}\right)\right\|_{2},
$$

and by induction we get the relation

$$
\sup _{z \in[0, L]}\left\|\delta \check{a}_{n}(z)\right\|_{2} \leq \frac{3 C^{2} L^{2}}{4} \sup _{z \in[0, L]}\left\|\delta \check{a}_{n-1}(z)\right\|_{2} .
$$

If $3 C^{2} L^{2}<4$, then $\delta \check{a}_{n}(z)$ converges at an exponential rate to 0 in $L^{\infty}\left([0, L], L^{2}\left(\mathbb{R}^{d}\right)\right)$, and therefore $\check{a}_{n}=\sum_{j=0}^{n-1} \delta \check{a}_{j}$ converges to a limit $\check{a}_{\infty}$. The same holds true for $\check{b}_{n}$ which converges to $\check{b}_{\infty}$. By taking the limit $n \rightarrow \infty$ in the integral formulation of the problem, we find that $\left(\check{a}_{\infty}, \breve{b}_{\infty}\right)$ is solution of the boundary value problem (14). Note that the limit problem has a unique solution, as can be shown by using the same arguments as above with the difference of two solutions.

We now prove the conservation of energy relation. We have

$$
\begin{aligned}
& \frac{d\left\|\check{b}_{\infty}(\omega, z)\right\|_{2}^{2}}{d z}=\Re\left(\frac{i \omega}{c_{0}} e^{-2 i \frac{\omega}{c_{0}} z} \int \nu(z, \mathbf{x}) \check{b}_{\infty}(\omega, z, \mathbf{x}) \check{a}_{\infty}(\omega, z, \mathbf{x}) d \mathbf{x}\right), \\
& \frac{d\left\|\check{a}_{\infty}(\omega, z)\right\|_{2}^{2}}{d z}=\Re\left(-\frac{i \omega}{c_{0}} e^{2 i \frac{\omega}{c_{0}} z} \int \nu(z, \mathbf{x}) \bar{a}_{\infty}(\omega, z, \mathbf{x}) \check{b}_{\infty}(\omega, z, \mathbf{x}) d \mathbf{x}\right),
\end{aligned}
$$

and therefore

$$
\frac{d\left\|\check{b}_{\infty}\right\|_{2}^{2}-\left\|\check{a}_{\infty}\right\|_{2}^{2}}{d z}=0 .
$$


Integrating this relation between $z=L$ and $z=0$ and using the boundary conditions, we obtain the conservation of energy relation.

Finally, for any $k \geq 1$, we have

$$
\left|\frac{d\left\|\delta \check{b}_{n}\right\|_{H^{k}}}{d z}\right| \leq C_{k}\left\|\delta \check{a}_{n-1}\right\|_{H^{k}}, \quad\left|\frac{d\left\|\delta \check{a}_{n}\right\|_{H^{k}}}{d z}\right| \leq C_{k}\left\|\delta \check{b}_{n}\right\|_{H^{k}}
$$

where

$$
C_{k}=\frac{\omega}{c_{0}} \sup _{z \in[0, L]}\|\nu(z, \cdot)\|_{W^{k, \infty}}
$$

We then proceed as above to get the final statement of Lemma 3.2.

\section{B Invariant Imbedding}

\section{B.1 General Theorem for a Two-point Linear Boundary Value Problem}

Let us consider the two-point boundary value problem:

$$
\frac{d X}{d z}(z)=\mathbf{A}(z) X(z), \quad X(z) \in \mathbb{R}^{m}
$$

with the boundary condition $\mathbf{H}^{0} X(0)+\mathbf{H}^{L} X(L)=V^{0}$, where $\mathbf{A}(z), \mathbf{H}^{0}$ and $\mathbf{H}^{L}$ are $m \times m$-matrices and $V^{0}$ is a $m$-dimensional vector. Assume that $\mathbf{A}(z)$ is bounded and $\mathbf{H}^{0}+\mathbf{H}^{L}$ is invertible. In this linear framework the invariant imbedding approach leads to the following proposition [3].

Proposition B.1 Let us define the matrices $(\mathbf{R}(\zeta))_{0 \leq \zeta \leq L}$ and $(\mathbf{Q}(z, \zeta))_{0 \leq z \leq \zeta \leq L}$ as the solutions of the initial value problems:

$$
\frac{d \mathbf{R}}{d \zeta}(\zeta)=\mathbf{A}(\zeta) \mathbf{R}(\zeta)-\mathbf{R}(\zeta) \mathbf{H}^{L} \mathbf{A}(\zeta) \mathbf{R}(\zeta), \quad 0 \leq \zeta \leq L
$$

starting from $\zeta=0: \mathbf{R}(\zeta=0)=\left(\mathbf{H}^{0}+\mathbf{H}^{L}\right)^{-1}$, and

$$
\frac{\partial \mathbf{Q}}{\partial \zeta}(z, \zeta)=-\mathbf{Q}(z, \zeta) \mathbf{H}^{L} \mathbf{A}(\zeta) \mathbf{R}(\zeta), \quad z \leq \zeta \leq L
$$

starting from $\zeta=z: \mathbf{Q}(z, \zeta=z)=\mathbf{R}(z)$. Then $\mathbf{P}(z)=\mathbf{Q}(z, L)$ is solution of:

$$
\frac{d \mathbf{P}}{d z}(z)=\mathbf{A}(z) \mathbf{P}(z), \quad 0 \leq z \leq L, \quad \text { with } \mathbf{H}^{0} \mathbf{P}(0)+\mathbf{H}^{L} \mathbf{P}(L)=\mathbf{I}
$$

and consequently $X(z)=\mathbf{Q}(z, L) V^{0}$ is solution of (B.1) with the boundary condition $\mathbf{H}^{0} X(0)+\mathbf{H}^{L} X(L)=V^{0}$. 
In particular, the value of $X(L)$ is $\mathbf{R}(L) V^{0}$. If we are interested in $X(L)$ only, then it is sufficient to consider the matrix Riccati equation (B.2).

\section{B.2 Application}

Let us fix the frequency $\omega$. We can apply the invariant imbedding theorem to the vector

$$
\widehat{\mathcal{X}}_{2 j-1}=\hat{a}_{j}, \quad \widehat{\mathcal{X}}_{2 j}=\hat{b}_{j}, \quad j=1, \ldots, n,
$$

with $\hat{a}_{j}$ and $\hat{b}_{j}$ defined by (20) and (19). It satisfies a two-point boundary value problem of the form

$$
\frac{d \widehat{\mathcal{X}}}{d z}=\widehat{\mathcal{A}}(z) \widehat{\mathcal{X}}, \quad z \in[0, L], \quad \widehat{\mathcal{H}}^{0} \widehat{\mathcal{X}}(0)+\widehat{\mathcal{H}}^{1} \widehat{\mathcal{X}}(L)=\widehat{\mathcal{V}}^{0}
$$

where the vector $\widehat{\mathcal{V}}^{0}$ is

$$
\widehat{\mathcal{V}}_{2 j-1}^{0}=0, \quad \widehat{\mathcal{V}}_{2 j}^{0}=\hat{b}_{\text {inc }}, \quad j=1, \ldots, n,
$$

and the elements of the operator matrices $\widehat{\mathcal{A}}(z), \widehat{\mathcal{H}}^{0}$, and $\widehat{\mathcal{H}}^{L}$ are zero apart from the following ones:

$$
\widehat{\mathcal{A}}_{2 j-1,2 j-1}(z)=\widehat{\mathcal{L}}(z), \quad \widehat{\mathcal{A}}_{2 j, 2 j}(z)=-\widehat{\mathcal{L}}(z), \quad \widehat{\mathcal{A}}_{2 j-1,2 j}(z)=\widehat{\mathcal{S}}^{-}(z),
$$

for $j=1, \ldots, n$,

$$
\widehat{\mathcal{A}}_{2 j, 2 j-3}(z)=-\widehat{\mathcal{S}}^{+}(z)
$$

for $j=2, \ldots, n$,

$$
\widehat{\mathcal{H}}_{2 j-1,2 j-1}^{0}=\widehat{\mathcal{I}}, \quad \widehat{\mathcal{H}}_{2 j, 2 j}^{L}=\widehat{\mathcal{I}},
$$

for $j=1, \ldots, n$, and the kernel of the operator $\hat{\mathcal{I}}$ is $\delta\left(\boldsymbol{\kappa}-\boldsymbol{\kappa}^{\prime}\right)$. By applying the general result, we first get that $\widehat{\boldsymbol{\mathcal { X }}}(L)=\widehat{\boldsymbol{\mathcal { R }}}(L) \widehat{\mathcal{V}}^{0}$ where $\widehat{\mathcal{\mathcal { R }}}$ is the operator matrix that satisfies the Riccati equation (B.2). After some algebra, we get the result as stated in (22-25). The operator $\widehat{\mathcal{R}}_{j}$ that appears in (23) is actually the component $\widehat{\boldsymbol{\mathcal { R }}}_{2 n-1,2 j}$ of the operator matrix $\widehat{\boldsymbol{\mathcal { R }}}$. Second, we get that $\widehat{\boldsymbol{\mathcal { X }}}(0)=$ $\widehat{\mathcal{Q}}(0, L) \widehat{\mathcal{V}}^{0}$ where $\widehat{\mathcal{Q}}$ is the operator matrix that satisfies equation (B.3). After some algebra, we get the result as stated in (29-30). The operator $\widehat{\mathcal{T}}_{j}$ that appears in (28) is actually the component $\widehat{\mathcal{Q}}_{2 n, 2 j}$ of the operator matrix $\widehat{\mathcal{Q}}$.

\section{References}

[1] M. Abramowitz and I. Stegun, Handbook of mathematical functions, Dover Publications, New-York, 1965. 
[2] G. Bal, G. Papanicolaou, and L. Ryzhik, Self-averaging in time reversal for the parabolic wave equation, Stochastics and Dynamics 2 (2002), 507-531.

[3] R. Bellman and G. M. Wing, Introduction to invariant imbedding, Wiley, New York, 1975.

[4] P. Blomgren, G. Papanicolaou, and H. Zhao, Super-resolution in time-reversal acoustics, J. Acoust. Soc. Am. 111 (2002), 230-248.

[5] M. D. Collins and R. B. Evans, A two-way parabolic equation for acoustic backscattering in the ocean, J. Acoust. Soc. Am. 91 (1992), 1357-1368.

[6] D. Dawson and G. Papanicolaou, A random wave process, Appl. Math. Optim. 12 (1984), 97-114.

[7] A. C. Fannjiang, White-noise and geometrical optics limits of Wigner-Moyal equation for beam waves in turbulent media II: Two-frequency formulation, J. Stat. Phys. 120 (2005), 543-586.

[8] A. Fannjiang and K. Sølna, Propagation and time-reversal of wave beams in atmospheric turbulence, SIAM Multiscale Model. Simul. 3 (2005), 522-558.

[9] J.-P. Fouque, J. Garnier, G. Papanicolaou, and K. Sølna, Wave propagation and time reversal in randomly layered media, Springer, New York, 2007.

[10] J. Garnier and K. Sølna, Random backscattering in the parabolic scaling, J. Stat. Phys. 131 (2008), 445-486.

[11] J. Garnier and K. Sølna, Background velocity estimation with cross correlations of incoherent waves in the parabolic scaling, submitted to Inverse Problems, available at http://www.math.uci.edu/ ksolna/

[12] K. Huang, K. Sølna, and H. Zhao, Coupled parabolic equations for wave propagation, Methods and Applications of Analysis 11 (2004), 399-412.

[13] K. Huang, G. Papanicolaou, K. Sølna, C. Tsogka, and H. Zhao, Efficient numerical simulation for long range wave propagation, J. Comput. Phys. 215 (2005), 448-464.

[14] G. Papanicolaou, L. Ryzhik, and K. Sølna, Self-averaging from lateral diversity in the Itô-Schrödinger equation, SIAM Multiscale Model. Simul. 6 (2007), 468492.

[15] J. W. Strohbehn, ed., Laser beam propagation in the atmosphere, Springer, Berlin, 1978. 